\title{
Electromagnetic propagators in hyperbolic Robertson-Walker cosmologies
}

\author{
Roman Tomaschitz ${ }^{\mathrm{a}}$ \\ Department of Physics, Hiroshima University, 1-3-1 Kagami-yama, \\ Higashi-Hiroshima 739-8526, Japan
}

(Received 15 August 2000; accepted for publication 5 September 2001)

\begin{abstract}
Green functions (retarded, advanced, Feynman and Dyson propagators) are calculated for the electromagnetic field in Robertson-Walker cosmologies with hyperbolic 3-manifolds as spacelike slices. The starting point is the Proca equation, i.e., the Maxwell field with a finite photon mass for infrared regularization, in a static cosmology with simply connected hyperbolic 3-sections. The time and space components of the resolvent kernel are scalar and vectorial point-pair invariants, respectively, and this symmetry allows for an explicit evaluation in the spectral representation. It is found that the quantum propagators have a logarithmic infrared singularity, which drops out in the zero curvature limit. Retarded and advanced Green functions remain well defined in the limit of zero photon mass, and they admit a simple generalization, by conformal scaling, to expanding 3-spaces. In cosmologies with multiply connected hyperbolic 3-manifolds as spacelike sections, the four enumerated propagators are constructed by means of Poincare series. The spectral decomposition of the Green functions is given in terms of Eisenstein series for a certain class of open hyperbolic 3-spaces, including those with Schottky covering groups corresponding to solid handle-bodies as spacelike slices. (C) 2001 American Institute of Physics. [DOI: 10.1063/1.1413522]
\end{abstract}

\section{INTRODUCTION}

This is a study of vectorial Green functions in hyperbolic Robertson-Walker (RW) cosmology. I will keep the introduction short, given the length of the paper, and given that it is a very technical paper too. No applications are discussed; the usefulness of Green functions in cosmology is documented in two standard reviews, ${ }^{1,2}$ which mainly focus on quantum field theory, and my motivation to write this paper is time-symmetric wave propagation in the cosmological absorber theory of Wheeler and Feynman, ${ }^{3,4}$ which, however, will not be addressed here. Electromagnetic Green functions in RW cosmologies with hyperbolic 3-sections have not been studied so far. There do exist a great many investigations on vector equations in Riemannian spaces, but they are either of a general nature, and hence not really explicit, or approximate, cf. the reviews cited above. I also note that the spaces considered in this paper are not asymptotically flat, and the high symmetry of the hyperbolic 3-space together with the conformal coupling of the electromagnetic field to the background geometry makes it possible to avoid the Riemann curvature tensor. Green functions for scalar fields on hyperbolic spaces, that is, the resolvent kernel of the LaplaceBeltrami operator, have been exhaustively studied ${ }^{5-10}$ (as far as one can go without specifying the covering group), but not so the resolvent kernels of vector operators, though, in two dimensions, there were attempts also in this regard. ${ }^{7,8}$

In Sec. II, the Proca equation is discussed (including spectral resolution and differential equations for the resolvent kernel) in a static RW cosmology with simply connected hyperbolic 3 -sections. This equation is tantamount to electrodynamics with a finite photon mass, ${ }^{11}$ but the mass parameter is regarded in this paper as a mere technical regularization of the Feynman and

${ }^{a)}$ Electronic mail: roman@fusion.sci.hiroshima-u.ac.jp 
Dyson propagators, which turn out to be infrared divergent, a curvature effect. In Sec. III, the longitudinal time component (a scalar point-pair invariant) of the resolvent kernel is calculated, as well as the time component $G_{00}$ of the real-space Green function, obtained by a Fourier transform. As in Minkowski space, the retarded and advanced Green functions and the two quantum propagators are defined by the choice of the integration path in the Fourier transform of the resolvent kernel.

In Sec. IV, the spatial resolvent kernel, a vectorial point-pair invariant, is calculated, and in Sec. V we study its Fourier transform, that is, the space components $G_{i j}$ of the Green function on the 4-manifold. (The space-time mixing components $G_{i 0}$ vanish for symmetry reasons.) Explicit expressions for all four real-space propagators are given, and their infrared behavior and the Minkowski space limit (curvature radius of the 3-space to infinity) is explained. At the end of Sec. $\mathrm{V}$, the time scaling of the two classical propagators (with zero photon mass) is discussed in RW cosmologies with arbitrary expansion factor.

In Sec. VI, we study Green functions on open, multiply connected hyperbolic manifolds (geometrically finite, without cusps) by periodizing the propagators calculated in the previous sections with the covering group. We focus on manifolds with $\delta(\Lambda)<1[\delta(\Lambda)$ : Hausdorff dimension of the limit set of the covering group], so that the Green functions can be defined by Poincare series without the use of analytic continuation. There are three appendices, all dealing with the calculation of matrix elements, which are reduced to convolutions of Poisson kernels and Feynman integrals. The orthogonality and completeness relations, and the various spectral measures used in Secs. III and IV are calculated there. The paper is more or less self-contained, the notation is mainly that of Ref. 5, which is also the approach to hyperbolic Green functions followed here.

\section{THE PROCA EQUATION IN A STATIC RW COSMOLOGY WITH NEGATIVELY CURVED 3-SECTIONS}

We consider a static RW line element, $d s^{2}=-d \tau^{2}+d \sigma^{2}$, where $d \sigma^{2}$ is the line element of hyperbolic space, corresponding to the metric $\gamma_{i j}=t^{-2} \delta_{i j}$ in the Poincare half-space model $H^{3}$ [with Cartesian coordinates $(z, t), t>0, z:=z_{1}+i z_{2}$ ], or to $\gamma_{i j}=4\left(1-|\mathbf{x}|^{2}\right)^{-2} \delta_{i j}$ in the ball model $B^{3}(|\mathbf{x}|<1)$, cf. Ref. 12. The field equations read

$$
F_{; \beta}^{\alpha \beta}+\mu^{2} A^{\alpha}=j^{\alpha},
$$

where $F_{\alpha \beta}=A_{\beta, \alpha}-A_{\alpha, \beta}$, and we have included a photon mass $\mu$ for the infrared regularization of quantum propagators. We find in $H^{3}$, with $x^{i}=\left(z_{1}, z_{2}, t\right)$,

$$
\begin{aligned}
& F_{0}{ }^{\beta} ; \beta=-t^{2} A_{0, n, n}+t A_{0,3}-t A_{3,0}+t^{2} A_{n, n, 0}, \\
& F_{i ; \beta}^{\beta} \equiv A_{i, 0,0}-t^{2} A_{i, n, n}+t A_{3, i}-t A_{i, 3}-A_{0,0, i}+t^{2} A_{n, n, i} .
\end{aligned}
$$

[Greek indices run from 0 to 3, Latin ones from 1 to 3, unless explicitly stated otherwise. Whenever a Latin subscript is attached twice to the same vector, ordinary summation is implied, e.g., in (2.2) and (2.3), summation over $n$.] In (2.1), we could substitute $F_{; \beta}^{\alpha \beta} \equiv-A_{; \beta}^{\alpha ; \beta}+R^{\alpha}{ }_{\beta} A^{\beta}$ $+A_{; \beta}^{\beta}{ }^{, \alpha}$, but it is better to avoid Christoffel symbols and the Ricci tensor.

The Lorentz condition, $A_{; \alpha}^{\alpha}=0$, is a consequence of (2.1) and current conservation, and we find, by differentiation,

$$
\begin{gathered}
-A_{0,0}+t^{2} A_{n, n}-t A_{3}=0, A_{0,0,0}-t^{2} A_{n, n, 0}+t A_{3,0}=0, \\
A_{0,0, i}-t^{2} A_{n, n, i}+t A_{3, i}-2 t \delta_{3 i} A_{n, n}+\delta_{3 i} A_{3}=0 .
\end{gathered}
$$

Using (2.4), we write the field Eqs. (2.1)-(2.3) as

$$
A_{0,0,0}-\Delta_{H^{3}} A_{0}+\mu^{2} A_{0}=j_{0}, \quad \Delta_{H^{3}}:=t^{2} \Delta_{E^{3}}-t \partial / \partial t,
$$




$$
A_{i, 0,0}-\Delta_{H^{3}} A_{i}-2 t\left(A_{i, 3}-A_{3, i}\right)-2 t \delta_{3 i} A_{n, n}+\delta_{3 i} A_{3}+\mu^{2} A_{i}=j_{i}
$$

where $\Delta_{H^{3}}$, is the scalar Laplace-Beltrami operator of $H^{3}$, and $\Delta_{E^{3}}$ is the Euclidean Laplace operator in Cartesian coordinates $x^{i}=\left(z_{1}, z_{2}, t\right)$. In this way we obtain separate equations for the space and time components of $A_{\alpha}$.

Analogously to (2.2) and (2.3), we find in the ball model $B^{3}$,

$$
\begin{gathered}
F_{0}{ }^{\beta} ; \beta \\
\equiv-(1 / 4)\left(1-|\mathbf{x}|^{2}\right)^{2}\left(A_{0, n, n}-A_{n, n, 0}\right)+(1 / 2)\left(1-|\mathbf{x}|^{2}\right) x^{n}\left(A_{n, 0}-A_{0, n}\right), \\
F_{i ; \beta}^{\beta} \equiv A_{i, 0,0}-A_{0,0, i}-(1 / 4)\left(1-|\mathbf{x}|^{2}\right)^{2}\left(A_{i, n, n}-A_{n, n, i}\right)-(1 / 2)\left(1-|\mathbf{x}|^{2}\right) x^{n}\left(A_{n, i}-A_{i, n}\right) .
\end{gathered}
$$

The Lorentz condition reads as

$$
-A_{0,0}+(1 / 2)\left(1-|\mathbf{x}|^{2}\right) x^{n} A_{n}+(1 / 4)\left(1-|\mathbf{x}|^{2}\right)^{2} A_{n, n}=0,
$$

to be differentiated with respect to space and time variables as in (2.4), and the separated field equations are

$$
\begin{gathered}
A_{0,0,0}-\Delta_{B^{3}} A_{0}+\mu^{2} A_{0}=j_{0}, \\
A_{i, 0,0}-\Delta_{B^{3}} A_{i}+(1 / 2)\left(1-|\mathbf{x}|^{2}\right)\left(2 x^{n}\left(A_{i, n}-A_{n, i}\right)-A_{i}+2 x_{i} A_{n, n}\right)+x_{i} x^{n} A_{n}+\mu^{2} A_{i}=j_{i}, \\
\Delta_{B^{3}}:=\frac{\left(1-|\mathbf{x}|^{2}\right)^{2}}{4}\left(\Delta_{E^{3}}+\frac{2}{1-|\mathbf{x}|^{2}} x_{n} \frac{\partial}{\partial x_{n}}\right) .
\end{gathered}
$$

In $H^{3}$, a set of transversal modes propagating along the $t$-semiaxis is readily found, Eqs. (2.4)-(2.6) (with $j_{\mu}=0$ ) are solved by

$$
A_{1}=t^{i s} e^{-i \omega \tau}, A_{0}=A_{2}=A_{3}=0, \omega^{2}=s^{2}+\mu^{2}
$$

and the same with $A_{1}$ and $A_{2}$ interchanged. The longitudinal modes traveling along the $t$-semiaxis read

$$
A_{3}=t^{i s} e^{-i \omega \tau}, A_{1}=A_{2}=0, A_{0}=-i(1-i s) \omega^{-1} t A_{3}, \omega^{2}=s^{2}+1+\mu^{2} .
$$

The time component $A_{0}$ is inferred from the Lorentz condition in (2.4), and one should also stress that the dispersion relation is different from the transversal modes.

A complete set of eigenfunctions can be generated by applying certain symmetry transformations of $H^{3}$ [Möbius transformations $\alpha_{\xi}(z)=(z-\xi)^{-1}$, lifted into $H^{3}$, cf. Ref. 12] to the plane waves (2.13) and (2.14). The dispersion relations remain unaltered, and we find, with $A_{\mu}$ $=\left(A_{0}, \mathbf{A}\right)$ and $i=1,2$, cf. Ref. 13,

$$
\begin{gathered}
A_{0}^{T_{i}}=0, \mathbf{A}^{T_{i}}(z, t ; \xi, s)=\mathbf{a}^{T_{i}}(z, t ; \xi, s) e^{-i \omega \tau}, \\
A_{0}^{L}(z, t ; \xi, s)=-i \omega^{-1}(1-i s) P^{1+i s}(z, t ; \xi) e^{-i \omega \tau}, \\
\mathbf{A}^{L}(z, t ; \xi, s)=\mathbf{a}^{L}(z, t ; \xi, s) e^{-i \omega \tau}, \\
\mathbf{a}^{L}(z, t ; \xi, s):=\frac{P^{2+i s}(z, t ; \xi)}{t^{2}}\left(\begin{array}{c}
-2 t\left(z_{1}-\xi_{1}\right) \\
-2 t\left(z_{2}-\xi_{2}\right) \\
|z-\xi|^{2}-t^{2}
\end{array}\right), P(z, t ; \xi):=\frac{t}{|z-\xi|^{2}+t^{2}},
\end{gathered}
$$




$$
\begin{aligned}
& \mathbf{a}^{T_{1}}(z, t ; \xi, s):=\frac{P^{2+i s}(z, t ; \xi)}{t^{2}}\left(\begin{array}{c}
-\left(z_{1}-\xi_{1}\right)^{2}+\left(z_{2}-\xi_{2}\right)^{2}+t^{2} \\
-2\left(z_{1}-\xi_{1}\right)\left(z_{2}-\xi_{2}\right) \\
-2 t\left(z_{1}-\xi_{1}\right)
\end{array}\right), \\
& \mathbf{a}^{T_{2}}(z, t ; \xi, s):=\frac{P^{2+i s}(z, t ; \xi)}{t^{2}}\left(\begin{array}{c}
-2\left(z_{1}-\xi_{1}\right)\left(z_{2}-\xi_{2}\right) \\
-\left(z_{2}-\xi_{2}\right)^{2}+\left(z_{1}-\xi_{1}\right)^{2}+t^{2} \\
-2 t\left(z_{2}-\xi_{2}\right)
\end{array}\right) .
\end{aligned}
$$

$\left[z:=\left(z_{1}, z_{2}\right), \xi:=\left(\xi_{1}, \xi_{2}\right) ;\right.$ it is useful to use complex notation as at the beginning of this section.] We have chosen the normalization $\mathbf{a}^{T_{i}}(z, t ; \xi, s) \cdot \mathbf{a}^{T_{i}}(z, t ; \xi,-s)=(P / t)^{2}$, and the same for the longitudinal component, and these vectors form an orthogonal triad; the $\mathbf{a}^{T_{1,2}}$ generate the tangent planes of the horospheres ${ }^{12} P(z, t ; \xi)=$ const., and $\mathbf{a}^{L}$ is proportional to the gradient of $P$.

The main part of this paper is based on the representation,

$$
\begin{gathered}
\mathbf{a}^{L}=\frac{1}{1+i s}\left(\begin{array}{c}
\frac{\partial P^{1+i s}}{\partial z_{1}} \\
\frac{\partial P^{1+i s}}{\partial z_{2}} \\
\frac{\partial P^{1+i s}}{\partial t}
\end{array}\right), \mathbf{a}^{T_{1}}=\frac{1}{1+i s}\left(\begin{array}{c}
\frac{-1}{4 i s} D_{12} P^{i s}+(1+i s) P^{2+i s} \\
\frac{-1}{2 i s} \frac{\partial^{2} P^{i s}}{\partial z_{1} \partial z_{2}} \\
\frac{\partial P^{1+i s}}{\partial z_{1}} \\
\frac{-1}{2 i s} \frac{\partial^{2} P^{i s}}{\partial z_{1} \partial z_{2}} \\
\mathbf{a}^{T_{2}}=\frac{1}{1+i s}\left(\begin{array}{c}
\frac{-1}{4 i s} D_{21} P^{i s}+(1+i s) P^{2+i s} \\
\frac{\partial P^{1+i s}}{\partial z_{2}}
\end{array}\right), D_{i j}:=\frac{\partial^{2}}{\partial z_{i}^{2}}-\frac{\partial^{2}}{\partial z_{j}^{2}},
\end{array}\right),
\end{gathered}
$$

and we will assume the identity,

$$
\frac{\partial P^{1+i s}}{\partial t}=(1+i s) P^{2+i s}\left(\frac{1}{t P}-2\right)
$$

substituted into the third component of $\mathbf{a}^{L}$, so that no $t$-derivatives appear in the eigenvectors. The $z_{i}$-derivatives in (2.18) may also be replaced by $\xi_{i}$-derivatives, via the substitution $\partial / \partial z_{i}$ $\rightarrow-\partial / \partial \xi_{i}$. When performing integrations over eigenfunctions, we will pull the differential operators in front of the integral signs, so that we are left with convolutions of Poisson kernels, which can be calculated by Feynman parametrization; this is the purpose of (2.18).

Finally, the eigenvectors (2.17) can be written as

$$
\begin{gathered}
\left(\mathbf{a}^{L}(z, t ; \xi, s)\right)^{t}=(0,0,1)\left[\alpha_{\xi}^{\prime}(z, t)\right] P^{i s}(z, t ; \xi), \\
\left(\mathbf{a}^{T_{1}}\right)^{t}=(1,0,0)\left[\alpha_{\xi}^{\prime}\right] P^{i s}, \quad\left(\mathbf{a}^{T_{2}}\right)^{t}=(0,-1,0)\left[\alpha_{\xi}^{\prime}\right] P^{i s},
\end{gathered}
$$

where $\left[\alpha_{\xi}^{\prime}\right]$ denotes the Jacobian of the Möbius transformation $\alpha_{\xi}(z)=(z-\xi)^{-1}$ lifted into the half-space. The superscript $t$ denotes transposition of the row-vectors (2.17), and ordinary matrix multiplication is assumed on the right-hand side of these equations. In Ref. 13, we derived

$$
\begin{gathered}
\left(\mathbf{a}^{L}(\gamma(z, t) ; \xi, s)\right)^{t}\left[\gamma^{\prime}(z, t)\right]=\left|\gamma^{-1 \prime} \xi\right|^{i s}\left(0,0,\left|\gamma^{-1 \prime} \xi\right|\right)\left[\alpha_{\gamma^{-1} \xi}^{\prime}(z, t)\right] P^{i s}\left(z, t ; \gamma^{-1} \xi\right), \\
\left(\mathbf{a}^{T_{1}}(\gamma ; \xi, s)\right)^{t}\left[\gamma^{\prime}\right]=\left|\gamma^{-1 \prime} \xi\right|^{i s}\left(\operatorname{Re}\left(\gamma^{-1 \prime} \xi\right),-\operatorname{Im}\left(\gamma^{-1 \prime} \xi\right), 0\right)\left[\alpha_{\gamma^{-1} \xi}^{\prime}(z, t)\right] P^{i s}\left(z, t ; \gamma^{-1} \xi\right),
\end{gathered}
$$




$$
\left(\mathbf{a}^{T_{2}}(\gamma ; \xi, s)\right)^{t}\left[\gamma^{\prime}\right]=\left|\gamma^{-1 \prime} \xi\right|^{i s}\left(-\operatorname{Im}\left(\gamma^{-1 \prime} \xi\right),-\operatorname{Re}\left(\gamma^{-1 \prime} \xi\right), 0\right)\left[\alpha_{\gamma^{-1}}^{\prime}(z, t)\right] P^{i s}\left(z, t ; \gamma^{-1} \xi\right),
$$

where $\gamma^{-1} \xi$ is the Möbius transformation $\gamma^{-1}$ acting in the complex plane, and $\gamma^{-1 \prime} \xi$ denotes its $\xi$-derivative. Introducing a circulary polarized basis for the transversal components,

$$
\begin{gathered}
\sqrt{2} \mathbf{a}^{T_{L}}:=\mathbf{a}^{T_{1}}+i \mathbf{a}^{T_{2}}, \quad \sqrt{2} \mathbf{a}^{T_{R}}:=\mathbf{a}^{T_{1}}-i \mathbf{a}^{T_{2}}, \\
\mathbf{a}^{T_{L}} \overline{\mathbf{a}^{T_{L}}}+\mathbf{a}^{T_{R}} \overline{\mathbf{a}^{T_{R}}}=\mathbf{a}^{T_{1}} \overline{\mathbf{a}^{T_{1}}}+\mathbf{a}^{T_{2}} \overline{\mathbf{a}^{T_{2}}},
\end{gathered}
$$

we find

$$
\begin{gathered}
{\left[\gamma^{\prime}(z, t)\right]^{t} \mathbf{a}^{L}(\gamma(z, t) ; \xi, s)=\left|\gamma^{-1 \prime} \xi\right|^{1+i s} \mathbf{a}^{L}\left(z, t ; \gamma^{-1} \xi, s\right),} \\
{\left[\gamma^{\prime}\right]^{t} \mathbf{a}^{T_{L}}(\gamma ; \xi)=\left|\gamma^{-1 \prime} \xi\right|^{i s} \overline{\gamma^{-1 \prime} \xi} \mathbf{a}^{T_{L}}\left(\gamma^{-1} \xi\right),} \\
{\left[\gamma^{\prime}\right]^{t} \mathbf{a}^{T_{R}}(\gamma ; \xi)=\left|\gamma^{-1 \prime} \xi\right|^{i s} \gamma^{-1 \prime} \xi \mathbf{a}^{T_{R}}\left(\gamma^{-1} \xi\right),}
\end{gathered}
$$

with $\left[\gamma^{\prime}\right]^{t} \mathbf{a}:=\mathbf{a}_{i}\left[\gamma^{\prime}\right]_{i j}$. ( $T_{L, R}$ stands for left and right transversal polarization, respectively.) As for the time component of the longitudinal modes in (2.16), we use $\mathrm{e}^{5,6}$

$$
P^{1+i s}(\gamma(z, t) ; \xi)=\left|\gamma^{-1 \prime} \xi\right|^{1+i s} P^{1+i s}\left(z, t ; \gamma^{-1} \xi\right),
$$

which is the scalar analog to (2.23). These symmetries are crucial in constructing the eigenfunctions of (2.5) and (2.6) on multiply connected manifolds, as well as the spectral representation of the resolvent, cf. Sec. VI.

Next we derive the differential equations for the propagators of the Proca Eq. (2.1). We start with a bivector $G_{\alpha \alpha^{\prime}}\left(x, x^{\prime}\right)$ on the 4-manifold; primed indices refer to the primed variable, and we define $F_{\alpha \alpha^{\prime} \beta}^{G}\left(x, x^{\prime}\right):=G_{\beta \alpha^{\prime} ; \alpha}-G_{\alpha \alpha^{\prime} ; \beta}$, which is evidently a second rank skew tensor with respect to $x$, and a vector with respect to $x^{\prime}$. The covariant differentiations may be replaced by ordinary ones, as usual in $F_{\alpha \beta}$. Likewise, in the divergence $F_{\alpha \alpha^{\prime} ; \beta}^{G}$, the primed index and the primed variable are regarded as dummy parameters in the differentiation procedure. Green functions are defined as solutions of

$$
F_{\alpha \alpha^{\prime} ; \beta}^{G \beta}+\mu^{2} G_{\alpha \alpha^{\prime}}=(-g)^{-1 / 2} \delta\left(x-x^{\prime}\right) g_{\alpha \alpha^{\prime}},
$$

$(-g)^{-1 / 2} \delta$ denotes the Dirac function on the 4-manifold. The variable in the metric and the determinant on the right-hand side may be $x$ or $x^{\prime}$, given the support of the $\delta$-function. Clearly, $G_{\alpha \alpha^{\prime}}\left(x, x^{\prime}\right)$ is an inverting kernel, since

$$
A_{\alpha}=\int G_{\alpha \alpha^{\prime}}\left(x, x^{\prime}\right) j^{\alpha^{\prime}}\left(x^{\prime}\right) \sqrt{-g\left(x^{\prime}\right)} d x^{\prime}
$$

solves the field Eq. (2.1) according to

$$
F_{\alpha ; \beta}^{\beta}+\mu^{2} A_{\alpha}=\int\left(F_{\alpha \alpha^{\prime} ; \beta}^{G}+\mu^{2} G_{\alpha \alpha^{\prime}}\right) j^{\alpha^{\prime}}\left(x^{\prime}\right) \sqrt{-g\left(x^{\prime}\right)} d x^{\prime}=j_{\alpha} .
$$

We consider, formally, the Fourier transform

$$
G_{\alpha \alpha^{\prime}}\left(\tau ; \mathbf{x}, \mathbf{x}^{\prime}\right)=(2 \pi)^{-1} \int_{-\infty}^{+\infty} d \omega e^{-i \omega \tau} \hat{G}_{\alpha \alpha^{\prime}}\left(\omega ; \mathbf{x}, \mathbf{x}^{\prime}\right),
$$

and find, with (2.10) and (2.11),

$$
-\Delta_{B^{3}} \hat{G}_{00}(\omega ; \mathbf{x}, \mathbf{y})+\left(\mu^{2}-\omega^{2}\right) \hat{G}_{00}=-(1 / 8)\left(1-|\mathbf{y}|^{2}\right)^{3} \delta(\mathbf{x}-\mathbf{y}),
$$




$$
\begin{aligned}
& -\Delta_{B^{3}} \hat{G}_{i j}(\omega ; \mathbf{x}, \mathbf{y})+(1 / 2)\left(1-|\mathbf{x}|^{2}\right)\left(2 x^{n}\left(\hat{G}_{i j, n}-\hat{G}_{n j, i}\right)-\hat{G}_{i j}+2 x_{i} \hat{G}_{n j, n}\right)+x_{i} x^{n} \hat{G}_{n j} \\
& \quad+\left(\mu^{2}-\omega^{2}\right) \hat{G}_{i j}=(1 / 2)\left(1-|\mathbf{y}|^{2}\right) \delta(\mathbf{x}-\mathbf{y}) .
\end{aligned}
$$

We have here slightly changed notation, writing $\mathbf{y}$ instead of $\mathbf{x}^{\prime}$, and all differentiations are with respect to the variable $\mathbf{x}$. In (2.28), we write as a shortcut $\tau$ instead of $\tau-\tau^{\prime} . \hat{G}_{00}$ and $\hat{G}_{i j}$ are scalar and vectorial point-pair invariants on the 3-space, respectively, cf. Ref. 13, and (6.3) and (6.4). (The first index always refers to $\mathbf{x}$ and the second to $\mathbf{y}$.) To achieve the symmetry $\hat{G}_{\mu \nu}(\omega ; \mathbf{x}, \mathbf{y})=\hat{G}_{\nu \mu}(\omega ; \mathbf{y}, \mathbf{x})$, we put the space-time mixing components to zero, that is $\hat{G}_{0 j}$ and $\hat{G}_{i 0}$, which satisfy the homogeneous Eqs. (2.29) and (2.30), respectively. Equations analogous to (2.29) and (2.30) hold in the half-space model $H^{3}$,

$$
\begin{gathered}
-\Delta_{H^{3}} \hat{G}_{00}\left(\omega ; z, t ; z_{0}, t_{0}\right)+\left(\mu^{2}-\omega^{2}\right) \hat{G}_{00}=-t^{3} \delta\left(t-t_{0}\right) \delta\left(z-z_{0}\right), \\
-\Delta_{H^{3}} \hat{G}_{i j}\left(\omega ; z, t ; z_{0}, t_{0}\right)-2 t\left(\hat{G}_{i j, 3}-\hat{G}_{3 j, i}\right)-2 t \delta_{3 i} \hat{G}_{n j, n}+\delta_{3 i} \hat{G}_{3 j}+\left(\mu^{2}-\omega^{2}\right) \hat{G}_{i j} \\
=t \delta\left(t-t_{0}\right) \delta\left(z-z_{0}\right) .
\end{gathered}
$$

[ $t$ is the coordinate in the half-space $H^{3}$ orthogonal to the complex plane; cosmic time is denoted by $\tau$.] In Secs. III and IV, we will solve (2.31) and (2.32), respectively, and figure out the boundary conditions, in terms of pole coefficient and decay at infinity. In Secs. II and V, we will discuss the Fourier transform (2.28), specify the integration paths encircling the singularities of the resolvent, and calculate the propagators, i.e., the general solution of Eq. (2.25).

\section{THE SCALAR TIME COMPONENT OF THE GREEN FUNCTION}

The differential equations for the time-time component $\hat{G}_{00}$ of the resolvent kernel only involve the Laplace-Beltrami operator (2.29) or (2.31), and accordingly $\hat{G}_{00}$ transforms as a scalar under spatial coordinate transformations. We put $\mathbf{y}=0$ in (2.29), define $\lambda:=\mu^{2}-\omega^{2}$ as spectral parameter, and look for spherically symmetric solutions $\psi(\lambda, r)$ of $\left(-\Delta_{B^{3}}+\lambda\right) \psi(\lambda, r)=0$ or

$$
\frac{-\left(1-r^{2}\right)^{2}}{4}\left[\psi^{\prime \prime}+2\left(\frac{1}{r}+\frac{r}{1-r^{2}}\right) \psi^{\prime}\right]+\lambda \psi=0
$$

Two independent solutions of (3.1) are

$$
\psi_{ \pm}(\lambda, r)=\left(1-r^{2}\right)^{1+\sqrt{1+\lambda}} r^{-1}\left((1+r)^{-2 \sqrt{1+\lambda}} \pm(1-r)^{-2 \sqrt{1+\lambda}}\right),
$$

and we assume $\operatorname{Re} \sqrt{1+\lambda}>0$. Since $\hat{G}_{00}(\omega ; \mathbf{x}, \mathbf{0})$ is spherically symmetric, it is a linear combination of the two solutions (3.2). As for boundary conditions, in the limit $r \rightarrow 0$, the asymptotic solution of Eq. (2.29) is $\hat{G}_{00}(\omega ; r, \mathbf{0}) \sim-1 /(8 \pi r)$, which follows from the Poisson equation $\Delta_{E^{3}} r^{-1}=-4 \pi \delta(\mathbf{x})$. The second integration constant is chosen in a way that $\hat{G}_{00}(\omega ; \mathbf{x}, \mathbf{0})$ decays as fast as possible at infinity, that is, for $r \rightarrow 1$. Accordingly,

$$
\hat{G}_{00}(\omega ; \mathbf{x}, \mathbf{0})=-\frac{1}{8 \pi r}\left(1-r^{2}\right)^{1+\sqrt{1+\lambda}}(1+r)^{-2 \sqrt{1+\lambda}} .
$$

The Green function with pole at $\mathbf{y}$ is obtained by the substitution ${ }^{5}$

$$
\mathbf{x} \rightarrow T_{\mathbf{y}} \mathbf{x}:=\frac{\left(1-|\mathbf{y}|^{2}\right) \mathbf{x}-\left(1-2 \mathbf{x} \cdot \mathbf{y}+|\mathbf{x}|^{2}\right) \mathbf{y}}{1-2 \mathbf{x} \cdot \mathbf{y}+|\mathbf{x}|^{2}|\mathbf{y}|^{2}} .
$$


$T_{\mathbf{y}}$ is a Möbius transformation in $B^{3}$ that maps an arbitrary point $\mathbf{y}$ into the origin. In fact, $\hat{G}_{00}\left(\omega ; T_{\mathbf{y}} \mathbf{x}, \mathbf{0}\right)$ is a solution of (2.29), denoted in the following by $\hat{G}_{00}(\omega ; \mathbf{x}, \mathbf{y})$, because (2.29) is invariant with respect to the symmetry group of $B^{3}$. The absolute value of (3.4) relates to the point-pair invariant ${ }^{5,12} L(\mathbf{x}, \mathbf{y})$ as

$$
\left|T_{\mathbf{y}} \mathbf{x}\right|=\sqrt{\frac{L(\mathbf{x}, \mathbf{y})}{1+L(\mathbf{x}, \mathbf{y})}}, \quad L(\mathbf{x}, \mathbf{y}):=\frac{|\mathbf{x}-\mathbf{y}|^{2}}{\left(1-|\mathbf{x}|^{2}\right)\left(1-|\mathbf{y}|^{2}\right)} .
$$

By substituting $r=\left|T_{\mathbf{y}} \mathbf{x}\right|$ into (3.3), we find the Green function with pole at $\mathbf{y}$, cf. Refs. 5, 6, and 10 ,

$$
\begin{gathered}
\hat{G}_{00}(\omega ; \mathbf{x}, \mathbf{y})=-\frac{1}{8 \pi} \frac{1}{\sqrt{L(1+L)}} \rho_{+}^{-\sqrt{1+\lambda}}(L)=-\frac{1}{4 \pi} \frac{e^{-\sqrt{1+\lambda} d}}{\sinh d}, \\
\rho_{ \pm}(L):=1+2 L \pm 2 \sqrt{L(L+1)}, \quad \rho_{+}=1 / \rho_{-}, \quad d:=\log \rho_{+}(L),
\end{gathered}
$$

with $\lambda=\mu^{2}-\omega^{2}$ and $\operatorname{Re} \sqrt{1+\lambda}>0$. We have here also introduced the hyperbolic distance function $d(\mathbf{x}, \mathbf{y})$ in $B^{3}$, and we will frequently make use of the identities,

$$
\begin{gathered}
(1 / 2)\left(\rho_{+}+\rho_{-}\right)=\cosh d=1+2 L, \\
(1 / 2)\left(\rho_{+}-\rho_{-}\right)=\sinh d=2 \sqrt{L(L+1)}, \\
e^{-d}=\rho_{-}=\frac{1-\sqrt{L /(1+L)}}{1+\sqrt{L /(1+L)}} .
\end{gathered}
$$

The Green function $\hat{G}_{00}\left(\omega ; z, t ; z_{0}, t_{0}\right)$ in the half-space model $H^{3}$, cf. (2.31), is obtained by the substitution,

$$
L(\mathbf{x}, \mathbf{y}) \rightarrow L\left(z, t ; z_{0}, t_{0}\right):=\frac{\left|z-z_{0}\right|^{2}+\left(t-t_{0}\right)^{2}}{4 t t_{0}}
$$

in (3.6) and (3.7). This follows from the isometry ${ }^{12} H^{3} \leftrightarrow B^{3}$,

$$
x^{i}=\left(|z|^{2}+(1+t)^{2}\right)^{-1}\left(2 z_{1}, 2 z_{2},|z|^{2}+t^{2}-1\right),
$$

with $z=z_{1}+i z_{2}$. The identities (3.6)-(3.10) also hold in $H^{3}$, with the distance function $d\left(z, t ; z_{0}, t_{0}\right)$ defined by the third equation in (3.7).

Next we compile the spectral representation ${ }^{6,10}$ of $\hat{G}_{00}\left(\omega ; z, t ; z_{0}, t_{0}\right)$,

$$
\begin{gathered}
\hat{G}_{00}\left(\omega ; z, t ; z_{0}, t_{0}\right)=-\int_{R^{2} \times R^{+}} d \sigma(\xi, s) \frac{P^{1+i s}(z, t ; \xi) P^{1-i s}\left(z_{0}, t_{0} ; \xi\right)}{s^{2}+1+\lambda} \\
=-\frac{1}{2 \pi^{3}} \int_{0}^{\infty} \frac{k(s, d)}{s^{2}+1+\lambda} s^{2} d s \\
k(s, d):=\int_{R^{2}} P^{1+i s}(z, t ; \xi) P^{1-i s}\left(z_{0}, t_{0} ; \xi\right) d \xi=\frac{\pi}{2} \frac{\sin \left(s \log \rho_{+}(L)\right)}{s \sqrt{L(1+L)}}=\pi \frac{\sin (s d)}{s \sinh (d)},
\end{gathered}
$$

with $\lambda(\omega)=\mu^{2}-\omega^{2}$. The spectral measure, $d \sigma(\xi, s)$, is defined in (A13) (with the convergence factor dropped). Equation (3.13) readily follows from the completeness relation (A14), in conjunction with $-\Delta_{H^{3}} P^{1+i s}=\left(s^{2}+1\right) P^{1+i s}$, cf. (B12), and (2.31). The integral in (3.14) is calcu- 
lated in (A7), and $\rho_{ \pm}(L)$ is defined in (3.7). As $\hat{G}_{00}$ depends on the space variables only via the point-pair invariant $L$ or the distance function $d$, we will write $\hat{G}_{00}(\omega, d)$; in this way we get model independent formulas, i.e., independent of the coordinate representation $H^{3}, B^{3}$, or any other.

Next we turn to the time dependent Green functions. The retarded Green function is denoted by $G_{00}^{+}$, the advanced one by $G_{00}^{-}$, the Feynman propagator by $G_{F 00}^{+}$, and the Dyson propagator by $G_{F 00}^{-}$; they can be defined by certain contour integrations, and we use the conventions, though not quite the notation, of Ref. 14. The propagators relate to $\hat{G}_{00}(\omega, d)$ in (3.6) via (2.28),

$$
\begin{gathered}
G_{(F) 00}^{ \pm}(\tau, d)=-\frac{1}{8 \pi^{2}} \int_{-\infty}^{+\infty} d \omega e^{-i \omega \tau} \frac{\exp \left(-\sqrt{1+\lambda_{(F)}^{ \pm}(\omega)} d\right)}{\sinh d}, \\
\lambda^{ \pm}(\omega):=\mu^{2}-\left(\omega^{2} \pm 2 i \varepsilon \omega\right), \quad \lambda_{F}^{ \pm}(\omega):=\mu^{2}-\left(\omega^{2} \pm i \varepsilon\right) .
\end{gathered}
$$

The $\varepsilon$-terms indicate the path of integration for the respective propagators. We find, via (3.13) and (3.14),

$$
\begin{gathered}
G_{(F) 00}^{ \pm}(\tau, d)=-\frac{1}{4 \pi^{4}} \int_{0}^{\infty} I_{(F)}^{ \pm}(s, \tau) k(s, d) s^{2} d s, \\
I_{(F)}^{ \pm}(s, \tau):=\int_{-\infty}^{+\infty} g_{(F)}^{ \pm}(s, \omega) \exp (-i \omega \tau) d \omega, \\
g^{ \pm}(s, \omega):=\left(\omega_{0}^{2}-\omega^{2} \mp 2 i \varepsilon \omega\right)^{-1}, \quad g_{F}^{ \pm}(s, \omega):=\left(\omega_{0}^{2}-\omega^{2} \mp i \varepsilon\right)^{-1},
\end{gathered}
$$

with $\omega_{0}(s):=\sqrt{s^{2}+1+\mu^{2}}$, and $\omega_{0}>0$. A standard integration ${ }^{14}$ gives

$$
I^{ \pm}(s, \tau)=\mp \pi i \omega_{0}^{-1}\left(e^{i \omega_{0} \tau}-e^{-i \omega_{0} \tau}\right) \theta( \pm \tau), \quad I_{F}^{ \pm}(s, \tau)= \pm \pi i \omega_{0}^{-1} e^{\mp i \omega_{0}|\tau|} .
$$

Defining

$$
\widetilde{G}_{00}(\tau, d):=-\frac{1}{4 \pi^{4}} \int_{0}^{\infty} \widetilde{I}(s, \tau, \varepsilon) k(s, d) s^{2} d s, \widetilde{I}(s, \tau, \varepsilon):=\omega_{0}^{-1} e^{-(i \tau+\varepsilon) \omega_{0}},
$$

we may write the propagators as linear combinations of $\widetilde{G}_{00}( \pm \tau, d)$,

$$
G_{00}^{ \pm}(\tau, d)= \pm \pi i \theta( \pm \tau)\left(\widetilde{G}_{00}(\tau, d)-\widetilde{G}_{00}(-\tau, d)\right), G_{F 00}^{ \pm}(\tau, d)= \pm \pi i \widetilde{G}_{00}( \pm|\tau|, d) .
$$

The integral in (3.19) is likewise standard, ${ }^{15}$ and we find

$$
\begin{gathered}
\widetilde{G}_{00}(\tau ; L)=-\frac{d}{4 \pi^{3}} \frac{\Delta(\tau, d)}{\sinh d}, \\
\Delta(\tau, d):=\frac{\sqrt{\mu^{2}+1} K_{1}\left(\sqrt{\mu^{2}+1} \sqrt{d^{2}-\tau^{2}+2 i \tau \varepsilon}\right)}{\sqrt{d^{2}-\tau^{2}+2 i \tau \varepsilon}} .
\end{gathered}
$$

Next we separate the pole part of $\Delta(\tau, d)$, and then perform the limit $\varepsilon \rightarrow 0$. We note $\mathrm{e}^{15}$

$$
\begin{gathered}
K_{1}( \pm i x)=-(\pi / 2)\left(J_{1}(x) \mp i N_{1}(x)\right), \quad x>0, \\
K_{1}(z)=z^{-1}+O(z \log z), \quad J_{1}(z)=z / 2+O\left(z^{3}\right), \\
N_{1}(z)=-(2 / \pi) z^{-1}+O(z \log z) .
\end{gathered}
$$


Hence,

$$
\begin{gathered}
\Delta(\tau, d)=\frac{1}{d^{2}-\tau^{2}+2 i \varepsilon \tau}-\frac{1}{d^{2}-\tau^{2}}+\hat{\delta}_{K}+\frac{\pi}{2}\left(\hat{\delta}_{N}+i \varepsilon(\tau) \hat{\delta}_{J}\right), \\
\hat{\delta}_{K}:=\theta\left(d^{2}-\tau^{2}\right) \sqrt{\mu^{2}+1} \frac{K_{1}\left(\sqrt{\mu^{2}+1} \sqrt{d^{2}-\tau^{2}}\right)}{\sqrt{d^{2}-\tau^{2}}}, \\
\hat{\delta}_{J, N}:=\theta\left(\tau^{2}-d^{2}\right) \sqrt{\mu^{2}+1} \frac{\left(J_{1}, N_{1}\right)\left(\sqrt{\mu^{2}+1} \sqrt{\tau^{2}-d^{2}}\right)}{\sqrt{\tau^{2}-d^{2}}},
\end{gathered}
$$

where $\varepsilon(\tau):=\operatorname{sign}(\tau)$ (not to be confused with the $\varepsilon$-regularization). The distributions (3.21) and (3.24) are identical. The second term in (3.24) is a principal value, and we arrive, by making use of the second formula in (A10), at

$$
\Delta(\tau, d)=-i \pi \delta\left(d^{2}-\tau^{2}\right) \varepsilon(\tau)+\hat{\delta}_{K}+(\pi / 2)\left(\hat{\delta}_{N}+i \varepsilon(\tau) \hat{\delta}_{J}\right)
$$

Piecing together the above formulas, we find the retarded/advanced Green functions and the Feynman/Dyson propagators as

$$
\begin{gathered}
G_{00}^{ \pm}(\tau, d)=-\frac{1}{2 \pi} \frac{d \theta( \pm \tau)}{\sinh d}\left(\delta\left(d^{2}-\tau^{2}\right)-\frac{1}{2} \hat{\delta}_{J}\right) \\
G_{F 00}^{ \pm}(\tau, d)=-\frac{1}{4 \pi} \frac{d}{\sinh d}\left(\delta\left(d^{2}-\tau^{2}\right)-\frac{1}{2} \hat{\delta}_{J} \pm \frac{i}{2}\left(\hat{\delta}_{N}+\frac{2}{\pi} \hat{\delta}_{K}\right)\right) .
\end{gathered}
$$

In the limit of vanishing photon mass, the classical propagators read

$$
\begin{aligned}
G_{00}^{ \pm}(\tau, d ; \mu=0)= & -\frac{1}{2 \pi} \frac{c^{2}}{R^{2}} \frac{d \theta( \pm \tau)}{\sinh (d / R)} \\
& \times\left(R \delta\left(d^{2}-c^{2} \tau^{2}\right)-\frac{1}{2} \frac{\theta\left(c^{2} \tau^{2}-d^{2}\right)}{\sqrt{c^{2} \tau^{2}-d^{2}}} J_{1}\left(R^{-1} \sqrt{c^{2} \tau^{2}-d^{2}}\right)\right) ;
\end{aligned}
$$

we have here restored the natural units, $R$ is the curvature radius of the 3 -space. The limit $\mu$ $\rightarrow 0$ can also be carried out in (3.29), but the spatial components of the quantum propagators get singular, see after (5.18).

Remark: In (2.1), (2.26), and (2.27), the restoration of the units requires the substitution $j^{\alpha}$ $\rightarrow c^{-1} j^{\alpha}$, and, for nonvanishing photon mass, $\mu:=m c / \hbar$. Moreover, $j^{0}=: \rho$ is the charge density of dimension $\sqrt{\hbar c} / R^{3}$. The components of the static RW-metric read in natural units $g_{00}=-c^{2}$ and $g_{i j}=(R / t)^{2} \delta_{i j}$, or $g_{i j}=4\left(1-|\mathbf{x}|^{2} / R^{2}\right)^{-2} \delta_{i j}, \quad|\mathbf{x}|<R$. Dimensionally, $j^{k} \sim c j^{0}, A^{k} \sim c A^{0}$ $\sim \sqrt{\hbar c} / R$, and $G_{00} \sim c^{2} G_{i j} \sim c^{2} / R^{2}$.

In the ball model $B^{3}$, the Minkowski space limit of (3.21) is recovered for $R \rightarrow \infty$. To this end, we restore the units in (3.21), analogously to (3.30). For $R \rightarrow \infty, d \sigma_{B^{3}}^{2} \sim 4 d \mathbf{x}^{2}$, so that $d \sim 2 \mid \mathbf{x}$ $-\mathbf{y} \mid$, and the asymptotic limit of the point-pair invariant (3.5) is $\sqrt{L} \sim R^{-1}|\mathbf{x}-\mathbf{y}|$. Also, $\mu^{2}+1$ $=(m c / \hbar)^{2}+R^{-2}$, see the Remark above. A rescaling of the space coordinates, $\mathbf{x} \rightarrow \mathbf{x} / 2$, then gives

$$
\widetilde{G}_{00}(\tau, d ; R \rightarrow \infty) \sim-\frac{\mu}{4 \pi^{3}} \frac{K_{1}\left(\mu \sqrt{|\mathbf{x}-\mathbf{y}|^{2}-\tau^{2}+2 i \tau \varepsilon}\right)}{\sqrt{|\mathbf{x}-\mathbf{y}|^{2}-\tau^{2}+2 i \tau \varepsilon}} .
$$

Inserting this into (3.28) and (3.29), we recover of course the Minkowski results. ${ }^{14}$ 
Remark: The Green functions $G_{(F) 00}^{ \pm}(\tau, d)$ also happen to be the propagators of the minimally coupled Klein-Gordon equation, solving

$$
\left(\square-\mu^{2}\right) G_{(F) 00}^{ \pm}(\tau, d(\mathbf{x}, \mathbf{y}))=c^{-1} \delta_{B^{3}}(\mathbf{x}, \mathbf{y}) \delta(\tau),
$$

with the d'Alembertian $\square=-\partial^{2} / \partial \tau^{2}+\Delta_{B^{3}}$. [The dimension of $G_{(F) 00}^{ \pm}$is here $1 / R^{2}$, so that we have to divide the right-hand side of (3.30) by $c^{2}$.] We may also couple the scalar field to the Ricci scalar, via the wave operator $\square-\mu^{2}-\xi \hat{R}$. As the background geometry is static, the Ricci scalar is constant, $\hat{R}=-6$, cf. (5.28). Obviously, $G_{(F) 00}^{ \pm}(\tau, d)$ also applies to this case, with a redefinition of the mass parameter, as long as $\mu^{2}+\xi \hat{R}+1 \geqslant 0$. Conformal coupling is achieved for $\mu=0$ and $\xi=1 / 6$. We will return to the conformally coupled Klein-Gordon equation, when we discuss electromagnetic propagators in an expanding background geometry, at the end of Sec. V.

\section{THE SPATIAL RESOLVENT KERNEL}

The transversal and longitudinal components of the spatial resolvent kernel, $\hat{R}_{i j}:=\hat{R}_{i j}^{T}+\hat{R}_{i j}^{L}$, of the Proca equation are defined by

$$
\begin{gathered}
\hat{R}_{i j}^{T}\left(z, t ; z^{\prime}, t^{\prime} ; \lambda\right):=\int_{R^{2} \times R^{+}} \sum_{k=1,2} \mathbf{a}_{i}^{T_{k}}(z, t ; \xi, s) \overline{\mathbf{a}_{j}^{T_{k}\left(z^{\prime}, t^{\prime} ; \xi, s\right)} \frac{d \sigma^{T}(\xi, s)}{s^{2}+\lambda},} \\
\hat{R}_{i j}^{L}\left(z, t ; z^{\prime}, t^{\prime} ; \lambda\right):=\int_{R^{2} \times R^{+}} \mathbf{a}_{i}^{L}(z, t ; \xi, s) \overline{\mathbf{a}_{j}^{L}\left(z^{\prime}, t^{\prime} ; \xi, s\right)} \frac{d \sigma^{L}(\xi, s)}{s^{2}+1+\lambda} .
\end{gathered}
$$

The eigenvectors $\mathbf{a}^{T_{1,2}, L}$ are listed in (2.18) and (2.19). In the spectral measures $d \sigma^{T, L}$, cf. (B13), the $\varepsilon$-regularizator can be dropped, as the integrals (4.1) and (4.2) are already convergent, unlike those in the completeness relation (B14). (As in Sec. III, we write the resolvent kernel with a roof, to distinguish it from its Fourier transform, when studying time-dependent Green functions on the 4-manifold in Sec. V.) If we put $\lambda=\mu^{2}-\omega^{2}$, then $\hat{R}_{i j}$ formally satisfies Eq. (2.32), which is an immediate consequence of the completeness relation (B.14). The vectorial space component of the (time independent) Green function of the Proca equation is thus

$$
\hat{G}_{i j}\left(\omega ; z, t ; z_{0}, t_{0}\right)=\hat{R}_{i j}\left(z, t ; z_{0}, t_{0} ; \lambda=\mu^{2}-\omega^{2}\right) .
$$

The time dependent Green function on the 4-manifold is then obtained by means of the Fourier transform (2.28), studied in Sec. V. The purpose of this section is to find an explicit formula for the kernel (4.3). A complex spectral parameter $\lambda$ is needed to define the integration paths in (2.28), which in turn define the propagators, cf. (3.17). In the following we assume $\operatorname{Re} \sqrt{\lambda}>0$, as well as $\operatorname{Re} \sqrt{\lambda+1}>0$.

At first we calculate the kernels (4.1) and (4.2) for $z=z^{\prime}=0$, and then restore these variables by a symmetry argument. To this end we need the matrix elements

$$
C_{i j}^{T_{k}}\left(t, t^{\prime} ; s\right):=\int_{R^{2}} \mathbf{a}_{i}^{T_{k}}(0, t ; \xi, s) \overline{\mathbf{a}_{j}^{T_{k}}\left(0, t^{\prime} ; \xi, s\right)} d \xi, \quad C_{i j}^{T}:=C_{i j}^{T_{1}}+C_{i j}^{T_{2}}, \quad C_{i j}^{L}:=\int_{R^{2}} \mathbf{a}_{i}^{L} \overline{\mathbf{a}_{j}^{L}} d \xi,
$$

calculated in Appendix C. The nonvanishing matrix elements of the kernels (at $z=z^{\prime}=0$ ) read, for $\beta=t^{\prime} / t>1$, 


$$
\begin{aligned}
& \hat{R}_{11}^{T}\left(0, t ; 0, t^{\prime} ; \lambda\right)=\int_{0}^{\infty} C_{11}^{T}\left(t, t^{\prime} ; s\right) \frac{s^{2}+1}{s^{2}+\lambda} \frac{d s}{2 \pi^{3}}=\frac{\beta}{2 \pi\left(\beta^{2}-1\right)^{3} \lambda} \frac{1}{t t^{\prime}}\left(4 \beta^{2}\left(\beta^{-\sqrt{\lambda}}-1\right)\right. \\
& \left.+\left(\beta^{4}-1\right) \sqrt{\lambda} \beta^{-\sqrt{\lambda}}+\left(\beta^{2}-1\right)^{2} \lambda \beta^{-\sqrt{\lambda}}\right), \\
& \hat{R}_{11}^{L}=\int_{0}^{\infty} C_{11}^{L}(s) \frac{s^{2}}{s^{2}+1+\lambda} \frac{d s}{2 \pi^{3}}=\frac{\beta^{2}}{\pi\left(\beta^{2}-1\right)^{3} \lambda} \frac{1}{t t^{\prime}} \\
& \times\left(2 \beta-\left(\beta^{2}+1\right) \beta^{-\sqrt{1+\lambda}}-\left(\beta^{2}-1\right) \sqrt{1+\lambda} \beta^{-\sqrt{1+\lambda}}\right), \\
& \hat{R}_{11}:=\hat{R}_{11}^{T}+\hat{R}_{11}^{L}=\frac{\beta}{2 \pi\left(\beta^{2}-1\right)^{3} \lambda} \frac{1}{t t^{\prime}}\left(4 \beta^{2-\sqrt{\lambda}}+\left(\beta^{4}-1\right) \sqrt{\lambda} \beta^{-\sqrt{\lambda}}+\left(\beta^{2}-1\right)^{2} \lambda \beta^{-\sqrt{\lambda}}\right. \\
& \left.-2 \beta\left(\beta^{2}+1\right) \beta^{-\sqrt{1+\lambda}}-2 \beta\left(\beta^{2}-1\right) \sqrt{1+\lambda} \beta^{-\sqrt{1+\lambda}}\right), \\
& \hat{R}_{22}^{(T, L)}=\hat{R}_{11}^{(T, L)}, \\
& \hat{R}_{33}^{T}:=\int_{0}^{\infty} C_{33}^{T}(s) \frac{s^{2}+1}{s^{2}+\lambda} \frac{d s}{2 \pi^{3}}=\frac{2 \beta^{2}}{\pi\left(\beta^{2}-1\right)^{3} \lambda} \frac{1}{t t^{\prime}} \\
& \times\left(\left(\beta^{2}+1\right)\left(1-\beta^{-\sqrt{\lambda}}\right)-\left(\beta^{2}-1\right) \sqrt{\lambda} \beta^{-\sqrt{\lambda}}\right), \\
& \hat{R}_{33}^{L}:=\int_{0}^{\infty} C_{33}^{L}(s) \frac{s^{2}}{s^{2}+1+\lambda} \frac{d s}{2 \pi^{3}}=\frac{\beta}{2 \pi\left(\beta^{2}-1\right)^{3} \lambda} \frac{1}{t t^{\prime}}\left(-4 \beta\left(\beta^{2}+1\right)+\left(\beta^{2}-1\right)^{2} \lambda \beta^{-\sqrt{1+\lambda}}\right. \\
& \left.+2\left(\beta^{4}-1\right) \sqrt{1+\lambda} \beta^{-\sqrt{1+\lambda}}+2\left(\beta^{2}+1\right)^{2} \beta^{-\sqrt{1+\lambda}}\right), \\
& \hat{R}_{33}:=\hat{R}_{33}^{T}+\hat{R}_{33}^{L}=\frac{\beta}{2 \pi\left(\beta^{2}-1\right)^{3} \lambda} \frac{1}{t t^{\prime}}\left(-4\left(\beta^{2}+1\right) \beta^{1-\sqrt{\lambda}}-4\left(\beta^{2}-1\right) \sqrt{\lambda} \beta^{1-\sqrt{\lambda}}\right. \\
& \left.+\left(\beta^{2}-1\right)^{2} \lambda \beta^{-\sqrt{1+\lambda}}+2\left(\beta^{4}-1\right) \sqrt{1+\lambda} \beta^{-\sqrt{1+\lambda}}+2\left(\beta^{2}+1\right)^{2} \beta^{-\sqrt{1+\lambda}}\right) .
\end{aligned}
$$

Replacing on the right-hand side of Eqs. (4.5)-(4.11) $\beta$ by $1 / \beta$, we obtain the kernels for $\beta$ $=t^{\prime} / t<1$. If we put $t^{\prime}=1$, and $t=1-2 r$, and calculate the leading order of $\hat{R}_{11}$ and $\hat{R}_{33}$ for $r$ $\rightarrow 0$, we find $\hat{R}_{11} \sim \hat{R}_{33} \sim 1 /(8 \pi r)$, and $\hat{R}_{11}^{L} \sim 1 /(16 \pi r)$.

Next we will restore the $z$ and $z^{\prime}$-variables, assumed zero in (4.5)-(4.11). This can be done by symmetry, of course. The goal is to find the Green function $\hat{G}_{i j}^{B^{3}}\left(\omega ; \mathbf{x}, \mathbf{x}^{\prime}\right)$ with pole at $\mathbf{x}^{\prime}$ in the Poincare ball $B^{3}$, cf. (2.30). [We will frequently switch between the ball $\left(B^{3}\right)$ and half-space $\left(H^{3}\right)$ models of hyperbolic geometry, cf. Sec. II, and we will indicate that by a superscript.] $\hat{G}_{i j}^{B^{3}}$ transforms as a bivector, $\hat{G}_{i j}^{B^{3}}\left(\omega ; \mathbf{x}, \mathbf{x}^{\prime}\right) d x^{i} d x^{\prime j}$; we map $B^{3}$ onto $H^{3}$ via the isometry (3.12), and use the same transformation for the primed coordinates $x^{\prime i}$ and $\left(z^{\prime}, t^{\prime}\right)$, so that $\mathbf{x}^{\prime}=0$ is mapped onto $\left(z^{\prime}=0, t^{\prime}=1\right)$. In the $B^{3}$-model, the Green function with pole at the origin is spherically symmetric, $\hat{G}_{i j}^{B^{3}}(\omega ; \mathbf{x}, \mathbf{0})=f(\omega ;|\mathbf{x}|) \delta_{i j}+g(\omega ;|\mathbf{x}|) x_{i} x_{j}$. We map $\hat{G}_{i j}^{B^{3}}\left(\omega ; \mathbf{x}, \mathbf{x}^{\prime}\right) d x^{i} d x^{\prime j}$ into $H^{3}$, and then consider the special coordinate values $\mathbf{x}^{\prime}=0, x^{1,2}=0, x^{3}=(t-1)(t+1)^{-1}, z=z^{\prime}=0, t^{\prime}$ $=1$, with arbitrary $t>0$. [From now on we also put $t^{\prime}=1$ in the components (4.5)-(4.11) of the resolvent kernel $\hat{R}_{i j}$, in particular $\beta=1 / t$.] At these special coordinate values, the result of this transformation is $\hat{R}_{11} d z_{1} d z_{1}^{\prime}+\hat{R}_{22} d z_{2} d z_{2}^{\prime}+\hat{R}_{33} d t d t^{\prime}$. [If $t>1$, we have to replace $\beta$ by $1 / \beta$ in (4.5)-(4.11), as mentioned above.] Moreover,

$$
d x^{i} d x^{\prime i}=(1+t)^{-2}\left(d z_{k} d z_{k}^{\prime}+d t d t^{\prime}\right), \quad x^{i} x^{j} d x^{i} d x^{\prime j}=\frac{(t-1)^{2}}{(t+1)^{4}} d t d t^{\prime}
$$


(We do not distinguish between $x^{i}$ and $x_{i}$; the $i$ and $j$-summations are from 1 to 3 , the $k$-summation is from 1 to 2 . Also note, that $x^{j}$ in the second formula is not primed.) Accordingly, we may identify

$$
f(\omega ;|\mathbf{x}|)=(1+t)^{2} \hat{R}_{11}, \quad g(\omega ;|\mathbf{x}|)=\frac{(1+t)^{4}}{(1-t)^{2}}\left(\hat{R}_{33}-\hat{R}_{11}\right),
$$

where $t=(1-|\mathbf{x}|)(1+|\mathbf{x}|)^{-1}$ if $t<1$, and $t=(1-|\mathbf{x}|)^{-1}(1+|\mathbf{x}|)$ if $t>1$. In this way we have determined the Green function $\hat{G}_{i j}^{B^{3}}(\omega ; \mathbf{x}, \mathbf{0})$ in $B^{3}$, with pole at the origin.

Remarks: We have put $t^{\prime}=1$ in $\hat{R}_{i j}$ for the $t$-dependence of $t \hat{R}_{i j}$ to be solely via $\beta=1 / t$. Equations (4.5)-(4.11) only hold for $\beta>1$, so that we have to choose $t=(1-|\mathbf{x}|)(1+|\mathbf{x}|)^{-1}$, if we use (4.7) and (4.11) in (4.13). As consistency check, the same result is obtained for $f(\omega ;|\mathbf{x}|)$ and $g(\omega ;|\mathbf{x}|)$, if we insert into (4.13) $\hat{R}_{i j}$ calculated for $\beta<1$ as indicated after (4.11). Then we have to identify $t=(1-|\mathbf{x}|)^{-1}(1+|\mathbf{x}|)$. As pointed out in (4.3), the spectral variable in $\hat{R}_{i j}$ relates to the frequency as $\lambda=\mu^{2}-\omega^{2}$.

Next we calculate, by a further symmetry argument, the Green function $\hat{G}_{i j}^{B^{3}}(\omega ; \mathbf{x}, \mathbf{y})$ in $B^{3}$ with pole at an arbitrary point $\mathbf{y}$. We write for the moment $\hat{\mathbf{x}}$ instead of $\mathbf{x}$, so that $\hat{G}_{i j}^{B^{3}}(\omega ; \hat{\mathbf{x}}, \mathbf{0})$ $=f(\omega ;|\hat{\mathbf{x}}|) \delta_{i j}+g(\omega ;|\hat{\mathbf{x}}|) \hat{x}_{i} \hat{x}_{j}$, which is to be regarded as a vector field depending on a dummy index $j$, solving the vector Eq. (2.30). Whenever we apply a Möbius transformation (i.e., a symmetry transformation of hyperbolic space) to this vector field, it will still solve the invariant Eq. (2.30). We consider the Möbius transformation (3.4), $\hat{\mathbf{x}}=T_{\mathbf{y}} \mathbf{x}$. The components of this transformation can be written in terms of the point-pair invariant $L(\mathbf{x}, \mathbf{y})$, cf. (3.5),

$$
\frac{\hat{x}_{j}}{1-|\mathbf{y}|^{2}}=-\frac{1}{2} \frac{1}{1+L} \frac{\partial L(\mathbf{x}, \mathbf{y})}{\partial y_{j}}
$$

and the Jacobian, $\hat{x}_{j, i}:=\partial \hat{x}_{j} / \partial x_{i}$, reads as

$$
\frac{\hat{x}_{j, i}}{1-|\mathbf{y}|^{2}}=\frac{1}{2} \frac{1}{(1+L)^{2}}\left(\frac{\partial L}{\partial x_{i}} \frac{\partial L}{\partial y_{j}}-(1+L) \frac{\partial^{2} L}{\partial x_{i} \partial y_{j}}\right) .
$$

By means of the identities,

$$
\begin{gathered}
|\hat{\mathbf{x}}|^{2}=\frac{L}{1+L}=\frac{|\mathbf{x}-\mathbf{y}|^{2}}{1-2 \mathbf{x} \cdot \mathbf{y}+|\mathbf{x}|^{2}|\mathbf{y}|^{2}} \\
\frac{1}{1+L} \frac{\partial L}{\partial y_{i}} \frac{\partial L}{\partial y_{i}}=\frac{4}{\left(1-|\mathbf{y}|^{2}\right)^{2}} L
\end{gathered}
$$

we readily find

$$
\frac{\hat{x}_{k} \hat{x}_{k, i} \hat{x}_{j}}{1-|\mathbf{y}|^{2}}=-\frac{1}{4} \frac{1}{(1+L)^{3}} \frac{\partial L}{\partial x_{i}} \frac{\partial L}{\partial y_{j}}
$$

[Summation over $i$ in (4.17) and $k$ in (4.18) is implied.] Applying the transformation $\hat{\mathbf{x}}=T_{\mathbf{y}} \mathbf{x}$ to $\hat{G}_{i j}^{B^{3}}(\omega ; \hat{\mathbf{x}}, \mathbf{0}) d \hat{x}_{i}$, we find

$$
\begin{gathered}
\hat{G}_{i j}^{B^{3}}(\omega ; \hat{\mathbf{x}}, \mathbf{0}) d \hat{x}_{i}=\left(1-|\mathbf{y}|^{2}\right) \hat{G}_{i j}^{B^{3}}(\omega ; \mathbf{x}, \mathbf{y}) d x_{i}, \\
\hat{G}_{i j}^{B^{3}}(\omega ; \mathbf{x}, \mathbf{y}):=f(\omega ;|\hat{\mathbf{x}}|) \frac{\hat{x}_{j, i}}{1-|\mathbf{y}|^{2}}+g(\omega ;|\hat{\mathbf{x}}|) \frac{\hat{x}_{k} \hat{x}_{k, i} \hat{x}_{j}}{1-|\mathbf{y}|^{2}},
\end{gathered}
$$

and more explicitly, by inserting (4.16), (4.15), and (4.18), 


$$
\hat{G}_{i j}^{B^{3}}(\omega ; \mathbf{x}, \mathbf{y})=\rho_{-} \hat{R}_{11}\left(\frac{(1+2 L)}{L(L+1)} L_{, i} L_{, j}-2 L_{, i, j}\right)-\rho_{-} \hat{R}_{33} \frac{1}{L(L+1)} L_{, i} L_{, j},
$$

with $\rho_{-}=1+2 L-2 \sqrt{L(L+1)}$ as defined in (3.7). The index $i$ denotes differentiation with respect to $x_{i}$, the index $j$ means differentiation with respect to $y_{j}$, and $\hat{R}_{11}$ and $\hat{R}_{33}$ are defined in (4.7) and (4.11), with $t^{\prime}=1$ and $t=1 / \beta=\rho_{-}=\sqrt{\rho_{-} / \rho_{+}}$. Note that $\hat{G}_{i j}^{B^{3}}(\omega ; \mathbf{x}, \mathbf{y})$ solely depends on the pointpair invariant $L(\mathbf{x}, \mathbf{y})$ and the spectral parameter $\lambda=\mu^{2}-\omega^{2}$ (via $\hat{R}_{11}$ and $\hat{R}_{33}$ ). In the definition (4.20), we have also used a normalization factor $\left(1-|\mathbf{y}|^{2}\right)^{-1}$, to achieve the symmetry $\hat{G}_{i j}^{B^{3}}(\omega ; \mathbf{x}, \mathbf{y})=\hat{G}_{j i}^{B^{3}}(\omega ; \mathbf{y}, \mathbf{x})$. By construction, $\hat{G}_{i j}^{B^{3}}(\omega ; \mathbf{x}, \mathbf{y})$ satisfies the vector Eq. (2.30), with the vector index $i$ corresponding to the argument $\mathbf{x} . \hat{G}_{i j}^{B^{3}}(\omega ; \mathbf{x}, \mathbf{y}) d x_{i} d y_{j}$ transforms as a bivector under arbitrary coordinate transformations $\mathbf{x}=h\left(\mathbf{x}^{\prime}\right), \mathbf{y}=h\left(\mathbf{y}^{\prime}\right)$, and $L(\mathbf{x}, \mathbf{y})$ is of course a biscalar. So it is clear how to map $\hat{G}_{i j}^{B^{3}}(\omega ; \mathbf{x}, \mathbf{y})$ into the $H^{3}$-model; we just have to replace in $(4.21) L(\mathbf{x}, \mathbf{y})$ by the $H^{3}$-point-pair invariant (3.11). [The biscalars $L(\mathbf{x}, \mathbf{y})$ and $L\left(z, t ; z^{\prime}, t^{\prime}\right)$ relate via the isometry (3.12).]

We also have to check that $\hat{G}_{i j}^{B^{3}}(\omega ; \mathbf{x}, \mathbf{y})$ has the right pole. We find in the $B^{3}$-model, from (4.21) and the discussion following (4.11),

$$
\hat{G}_{i j}^{B^{3}}(\omega ; \mathbf{x , y}) \sim \frac{1}{2 \pi} \frac{1}{1-|\mathbf{y}|^{2}} \frac{1}{|\mathbf{x}-\mathbf{y}|} \delta_{i j}
$$

which solves

$$
-\frac{1}{4}\left(1-|\mathbf{x}|^{2}\right)^{2} \Delta_{E^{3}} \hat{G}_{i j}^{B^{3}}=\gamma_{i j} \frac{1}{\sqrt{\gamma}} \delta(\mathbf{x}-\mathbf{y}),
$$

where $\gamma_{i j}=4\left(1-|\mathbf{x}|^{2}\right)^{-2} \delta_{i j}$ is the $B^{3}$-metric and $\gamma$ is its determinant. In fact, Eq. (2.30) boils down to (4.23) in leading asymptotic order.

We rewrite $\hat{G}_{i j}^{B^{3}}(\omega ; \mathbf{x}, \mathbf{y})$ in terms of the hyperbolic distance function $d(\mathbf{x}, \mathbf{y})$, cf. (3.8)-(3.10). As in (4.21), the indices $i$ and $j$ denote differentiation with respect to the first and second argument, respectively. By differentiating Eq. (3.8), we readily find

$$
d_{, i} d_{, j} \sinh ^{2} d=4 L_{, i} L_{, j}, \quad d_{, i} d_{, j} \cosh d+d_{, i, j} \sinh d=2 L_{, i, j} .
$$

We may now write, instead of (4.21),

$$
\hat{G}_{i j}(\omega ; \mathbf{x}, \mathbf{y})=-e^{-d}\left(\hat{R}_{11} \sinh d d_{, i, j}+\hat{R}_{33} d_{, i} d_{, j}\right),
$$

and in (4.7) and (4.11) we put $t^{\prime}=1$ and $t=1 / \beta=e^{-d}$, so that

$$
\begin{gathered}
\hat{R}_{11}=\frac{e^{d}}{4 \pi \lambda \sinh ^{3} d}\left(e^{-\sqrt{\lambda} d}\left(1+\lambda \sinh ^{2} d+\sqrt{\lambda} \sinh d \cosh d\right)-e^{-\sqrt{1+\lambda} d}(\cosh d+\sqrt{1+\lambda} \sinh d)\right) \\
\hat{R}_{33}=\frac{-e^{d}}{2 \pi \lambda \sinh ^{3} d}\left(e^{-\sqrt{\lambda} d}(\cosh d+\sqrt{\lambda} \sinh d)-e^{-\sqrt{1+\lambda} d}\left(\cosh ^{2} d+(\lambda / 2) \sinh ^{2} d\right.\right. \\
+\sqrt{1+\lambda} \sinh d \cosh d))
\end{gathered}
$$

with $\operatorname{Re} \sqrt{\lambda}>0$, and $\operatorname{Re} \sqrt{\lambda+1}>0$. The Green function $\hat{G}_{i j}(\omega ; \mathbf{x}, \mathbf{y})$, explicitly defined by (4.25)(4.27) depends on the space arguments only via the hyperbolic distance function $d(\mathbf{x}, \mathbf{y})$. We have dropped the $B^{3}$-superscript, as Eqs. (4.25)-(4.27) are valid in every model of hyperbolic geom- 
etry, e.g., in the half-space model $H^{3}$, where $d(\mathbf{x}, \mathbf{y})$ is to be replaced by the $H^{3}$-distance function $d\left(z, t ; z_{0}, t_{0}\right)$, defined by (3.10) and (3.11). As special case, we note the Green function (4.25) with pole at $\mathbf{y}=0$ in the $B^{3}$-model,

$$
\hat{G}_{i j}^{B^{3}}(\omega ;|\mathbf{x}|, \mathbf{0})=\frac{4 \hat{R}_{11}}{(1+r)^{2}} \delta_{i j}+\frac{4\left(\hat{R}_{33}-\hat{R}_{11}\right)}{(1+r)^{2}} \frac{x_{i} x_{j}}{r^{2}},
$$

where $\hat{R}_{11}$ and $\hat{R}_{33}$ are defined by (4.26) and (4.27) and the substitutions

$$
\sinh d=\frac{2 r}{1-r^{2}}, \quad \cosh d=\frac{1+r^{2}}{1-r^{2}}, \quad e^{d}=\frac{1+r}{1-r},
$$

cf. (3.5) and (3.8)-(3.10).

Finally we give a direct proof of the completeness relation (B14), which is a good consistency check for the rather lengthy calculations in this section and Appendix C. We start with (4.25), but use for $\hat{R}_{11}$ and $\hat{R}_{33}$ the integral representations in (4.1) and (4.2), with the $\left(s^{2}+\lambda\right)^{-1}$ and $\left(s^{2}\right.$

$+1+\lambda)^{-1}$ factors dropped. Instead, we include into these integrals the convergence factor $e^{-\varepsilon s}$ that appears in the spectral measures (B13). In short, by replacing in (4.25) $\hat{R}_{i j}$ by

$$
\hat{S}_{i j}:=\frac{1}{2 \pi^{3}} \int_{0}^{\infty} d s e^{-\varepsilon s}\left(\left(s^{2}+1\right) C_{i j}^{T}(s)+s^{2} C_{i j}^{L}(s)\right),
$$

cf. (4.4) and (C.8), we may write the completeness relation (B14) as

$$
-e^{-d}\left(\hat{S}_{11} \sinh d d_{, i, j}+\hat{S}_{33} d_{, i} d_{, j}\right)=\gamma_{i j} \gamma^{-1 / 2} \delta(\mathbf{x}-\mathbf{y}) .
$$

It is here understood, that the substitutions $t^{\prime}=1$ and $t=1 / \beta=e^{-d}$, as indicated after (4.25), are carried out in the $C_{i j}^{T, L}$-coefficients. It is not difficult to verify (4.31). The various $s$-integrations in $\hat{S}_{11}$ and $\hat{S}_{33}$ are all standard in the limit $\varepsilon \rightarrow 0$. [We put $\varepsilon=0$ whenever the integrals over the individual trigonometric functions in the $C_{i j}^{T, L}$-coefficients converge without regularization, cf. (C8).] We find, with $d \sim 2 \sqrt{L}$ for $\mathbf{x} \rightarrow \mathbf{y}$,

$$
\hat{S}_{11}=4 \pi \frac{\beta^{2} \log \beta}{\beta^{2}-1} \frac{\varepsilon}{\left(\varepsilon^{2}+\log ^{2} \beta\right)^{2}}=\frac{1}{\pi^{2}} \frac{\varepsilon}{\left(\varepsilon^{2}+4 L\right)^{2}},
$$

which is an $\varepsilon$-representation of the $\delta$-function, $\gamma^{-1 / 2} \delta(\mathbf{x}-\mathbf{y})$, in hyperbolic space, cf. (A16). Moreover, at $\mathbf{x}=\mathbf{y}$,

$$
L_{, i, j}=\frac{-2}{\left(1-|\mathbf{y}|^{2}\right)^{2}} \delta_{i j}+O\left(x_{i} x_{j}\right), L_{, i} L_{, j}=O\left(x_{i} x_{j}\right),
$$

and it is easy to check, that the $O\left(x_{i} x_{j}\right)$-terms entering in (4.31) via (4.24) do not give a contribution for $\varepsilon \rightarrow 0$, which proves (4.31) and hence the completeness relation (B14).

\section{TIME DEPENDENT GREEN FUNCTIONS}

In this section we focus on the Fourier integral (2.28) for the spatial component of the Green function, calculated in (4.25)-(4.27). The time component of the Green function has already been Fourier transformed in Sec. III, cf. (3.15)-(3.30), and a similar procedure is applied in this section to the spatial Fourier component (4.25). The integration paths in (2.28) are specified by substituting for $\lambda$ in (4.25) one of the four $\varepsilon$-regularizations $\lambda_{(F)}^{ \pm}(\omega)$ defined in (3.16). $\lambda^{ \pm}$refers to 
retarded $(+)$ and advanced Green functions, and $\lambda_{F}^{ \pm}$to Feynman $(+)$and Dyson propagators. Also note that we assumed in the derivation of (4.25) $\operatorname{Re} \sqrt{\lambda}>0$, and $\operatorname{Re} \sqrt{\lambda+1}>0$. We write the matrix elements (4.26) and (4.27) as

$$
\begin{aligned}
& \hat{R}_{11}(\lambda, d)= \frac{e^{d}}{4 \pi \sinh ^{3} d}\left(-\int_{0}^{d} \frac{e^{-\sqrt{\lambda} x}}{\sqrt{\lambda}} d x+\frac{e^{-\sqrt{\lambda} d}}{\sqrt{\lambda}} \sinh d \cosh d+e^{-\sqrt{\lambda} d} \sinh ^{2} d\right. \\
&\left.+\int_{0}^{d} e^{-\sqrt{1+\lambda} x} \sinh x d x\right), \\
& \hat{R}_{33}(\lambda, d)= \frac{e^{d}}{2 \pi \sinh ^{3} d}\left(\cosh d \int_{0}^{d} \frac{e^{-\sqrt{\lambda} x}}{\sqrt{\lambda}} d x-\frac{e^{-\sqrt{\lambda} d}}{\sqrt{\lambda}} \sinh d-\cosh d \int_{0}^{d} e^{-\sqrt{1+\lambda} x} \sinh x d x\right. \\
&\left.+\frac{1}{2} e^{-\sqrt{1+\lambda} d} \sinh ^{2} d\right),
\end{aligned}
$$

and substitute

$$
\frac{e^{-\sqrt{\lambda} d}}{\sqrt{\lambda}}=\frac{2}{\pi} \int_{0}^{\infty} \frac{\cos (s d)}{s^{2}+\lambda} d s, e^{-\sqrt{\lambda} d}=\frac{2}{\pi} \int_{0}^{\infty} \frac{s \sin (s d)}{s^{2}+\lambda} d s,
$$

and the same with $\lambda \rightarrow \lambda+1$, and $d \rightarrow x$. (We do not interchange the $s$ and $d$-integrations here.) Next we define,

$$
R_{(F) i j}^{ \pm}(\tau, d):=\frac{1}{2 \pi} \int_{-\infty}^{\infty} d \omega e^{-i \omega \tau} \hat{R}_{i j}\left(\lambda_{(F)}^{ \pm}(\omega), d\right),
$$

so that retarded and advanced Green functions and Feynman and Dyson propagators read, according to $(4.25)$,

$$
G_{(F) i j}^{ \pm}(\tau ; \mathbf{x}, \mathbf{y}):=-e^{-d}\left(R_{(F) 11}^{ \pm}(\tau, d) \sinh d d_{, i, j}+R_{(F) 33}^{ \pm}(\tau, d) d_{, i} d_{, j}\right) .
$$

This complements the time component $G_{(F) 00}^{ \pm}(\tau, d)$ in (3.15). To evaluate (5.4), we interchange $s$ and $\omega$-integrations. The $\omega$-integration can be carried out as in (3.17) and (3.18), and we arrive at

$$
\begin{aligned}
R_{(F) 11}^{ \pm}(\tau, d)= & \frac{e^{d}}{4 \pi \sinh ^{3} d}\left(-\int_{0}^{d} S_{0(F)}^{ \pm}(\tau, x ; \mu) d x+S_{0(F)}^{ \pm}(\tau, d ; \mu) \sinh d \cosh d\right. \\
& \left.+S_{1(F)}^{ \pm}(\tau, d ; \mu) \sinh ^{2} d+\int_{0}^{d} S_{1(F)}^{ \pm}\left(\tau, x ; \sqrt{1+\mu^{2}}\right) \sinh x d x\right), \\
R_{(F) 33}^{ \pm}(\tau, d)= & \frac{e^{d}}{2 \pi \sinh ^{3} d}\left(\cosh d \int_{0}^{d} S_{0(F)}^{ \pm}(\tau, x ; \mu) d x-S_{0(F)}^{ \pm}(\tau, d ; \mu) \sinh d\right. \\
& \left.-\cosh d \int_{0}^{d} S_{1(F)}^{ \pm}\left(\tau, x ; \sqrt{1+\mu^{2}}\right) \sinh x d x+\frac{1}{2} S_{1(F)}^{ \pm}\left(\tau, d ; \sqrt{1+\mu^{2}}\right) \sinh ^{2} d\right) .
\end{aligned}
$$

Here, $S_{i(F)}^{ \pm}(\tau, d ; \mu)$ and $S_{i(F)}^{ \pm}\left(\tau, d ; \sqrt{1+\mu^{2}}\right)$ are defined by

$$
S_{0(F)}^{ \pm}(\tau, d ; \mu):=\frac{1}{\pi^{2}} \int_{0}^{\infty} \cos (s d) I_{(F)}^{ \pm}\left(\omega_{0}, \tau\right) d s,
$$




$$
\begin{gathered}
S_{1(F)}^{ \pm}(\tau, d ; \mu):=\frac{1}{\pi^{2}} \int_{0}^{\infty} s \sin (s d) I_{(F)}^{ \pm}\left(\omega_{0}, \tau\right) d s, \\
I^{ \pm}\left(\omega_{0}, \tau\right):=\mp \pi i \omega_{0}^{-1}\left(e^{i \omega_{0} \tau}-e^{-i \omega_{0} \tau}\right) \theta( \pm \tau), I_{F}^{ \pm}\left(\omega_{0}, \tau\right):= \pm \pi i \omega_{0}^{-1} e^{\mp i \omega_{0}|\tau|},
\end{gathered}
$$

with $\omega_{0}:=\sqrt{s^{2}+\mu^{2}}$, and Re $\omega_{0}>0$. Sometimes we will write $\omega_{0}^{T}$ for $\omega_{0}$, the frequency of the transversal modes; the longitudinal frequency relates to the spectral parameter via $\omega_{0}^{L}$ $:=\sqrt{s^{2}+1+\mu^{2}}$, Re $\omega_{0}^{L}>0$. Evidently, $S_{i(F)}^{ \pm}\left(\tau, d ; \sqrt{1+\mu^{2}}\right)$ is obtained by replacing in (5.8) $\omega_{0}$ by $\omega_{0}^{L}$. The terms in (5.6) and (5.7) depending on $S_{i(F)}^{ \pm}(\tau, d ; \mu)$ stem from the transversal modes in the spectral resolution, cf. the derivation of (4.26) and (4.27), whereas the terms containing $S_{i(F)}^{ \pm}\left(\tau, d ; \sqrt{1+\mu^{2}}\right)$ are generated by the longitudinal modes. Clearly, $S_{1}=-\partial S_{0} / \partial d$. The coefficients (5.8) are calculated via an integral representation of modified Bessel functions, ${ }^{15}$ quite analogously to the scalar case (3.19) and (3.20),

$$
\begin{gathered}
S_{i}^{ \pm}(\tau, d ; \mu)= \pm \pi i\left(\widetilde{S}_{i}(\tau, d ; \mu)-\widetilde{S}_{i}(-\tau, d ; \mu)\right) \theta( \pm \tau), \\
S_{i F}^{ \pm}(\tau, d ; \mu)= \pm \pi i \widetilde{S}_{i}( \pm|\tau|, d ; \mu),
\end{gathered}
$$

where we have defined

$$
\begin{gathered}
\widetilde{S}_{0}(\tau, d ; \mu):=\frac{1}{\pi^{2}} \int_{0}^{\infty} \cos (s d) \widetilde{I}\left(\omega_{0}, \tau\right) d s=\frac{1}{\pi^{2}} K_{0}\left(\mu \sqrt{d^{2}+(i \tau+\varepsilon)^{2}}\right), \\
\widetilde{S}_{1}(\tau, d ; \mu):=\frac{1}{\pi^{2}} \int_{0}^{\infty} s \sin (s d) \widetilde{I}\left(\omega_{0}, \tau\right) d s=\frac{\mu d}{\pi^{2}} \frac{K_{1}\left(\mu \sqrt{d^{2}+(i \tau+\varepsilon)^{2}}\right)}{\sqrt{d^{2}+(i \tau+\varepsilon)^{2}}},
\end{gathered}
$$

with $\widetilde{I}\left(\omega_{0}, \tau\right):=\omega_{0}^{-1} e^{-(i \tau+\varepsilon) \omega_{0}}$, and the same with $\mu \rightarrow \sqrt{1+\mu^{2}}$. Performing the limit $\varepsilon \rightarrow 0$, cf. (3.21)-(3.27), we find

$$
\begin{gathered}
\widetilde{S}_{0}(\tau, d ; \mu)=\frac{1}{\pi^{2}} \theta\left(d^{2}-\tau^{2}\right) K_{0}\left(\mu \sqrt{d^{2}-\tau^{2}}\right)-\frac{1}{2 \pi} \theta\left(\tau^{2}-d^{2}\right) \\
\times\left(N_{0}\left(\mu \sqrt{\tau^{2}-d^{2}}\right)+i \varepsilon(\tau) J_{0}\left(\mu \sqrt{\tau^{2}-d^{2}}\right)\right), \\
\widetilde{S}_{1}(\tau, d ; \mu)=-\frac{i}{\pi} d \varepsilon(\tau) \delta\left(d^{2}-\tau^{2}\right)+\frac{\mu d}{\pi^{2}} \frac{\theta\left(d^{2}-\tau^{2}\right)}{\sqrt{d^{2}-\tau^{2}}} K_{1}\left(\mu \sqrt{d^{2}-\tau^{2}}\right) \\
+\frac{\mu d}{2 \pi} \frac{\theta\left(\tau^{2}-d^{2}\right)}{\sqrt{\tau^{2}-d^{2}}}\left(N_{1}\left(\mu \sqrt{\tau^{2}-d^{2}}\right)+i \varepsilon(\tau) J_{1}\left(\mu \sqrt{\tau^{2}-d^{2}}\right)\right),
\end{gathered}
$$

and the $\widetilde{S}_{i}\left(\tau, d ; \sqrt{1+\mu^{2}}\right)$ are obtained by the substitution $\mu \rightarrow \sqrt{1+\mu^{2}}$, of course; $\varepsilon(\tau)$ is the sign function. In the calculation of (5.13) and (5.14), we used the second and third formula in (A10), as well as (3.22) and (3.23), and ${ }^{15}$

$$
\begin{gathered}
K_{0}( \pm i x)=-(\pi / 2)\left(N_{0}(x) \pm i J_{0}(x)\right), \quad x>0, \\
K_{0}(z)=-\gamma-\log (z / 2)+O\left(z^{2} \log z\right), \quad J_{0}(z)=1+O\left(z^{2}\right), \\
N_{0}(z)=(2 / \pi)(\gamma+\log (z / 2))+O\left(z^{2} \log z\right) .
\end{gathered}
$$

The integrals in (5.6) and (5.7) are calculated via (5.9) and (5.10), by making use of (5.13) and (5.14), 


$$
\begin{aligned}
& \int_{0}^{d} \widetilde{S}_{0}(\tau, x ; \mu) d x=\frac{1}{\pi^{2}} \theta\left(d^{2}-\tau^{2}\right) \int_{0}^{\sqrt{d^{2}-\tau^{2}}} \frac{K_{0}(\mu y) y d y}{\sqrt{\tau^{2}+y^{2}}}-\frac{1}{2 \pi}\left(\theta\left(\tau^{2}-d^{2}\right) \int_{\sqrt{\tau^{2}-d^{2}}}^{|\tau|}\right. \\
& \left.+\theta\left(d^{2}-\tau^{2}\right) \int_{0}^{|\tau|}\right)\left(N_{0}(\mu y)+i \varepsilon(\tau) J_{0}(\mu y)\right) \frac{y d y}{\sqrt{\tau^{2}-y^{2}}}, \\
& \int_{0}^{d} \widetilde{S}_{1}\left(\tau, x ; \sqrt{1+\mu^{2}}\right) \sinh x d x=-\frac{i}{2 \pi} \theta\left(d^{2}-\tau^{2}\right) \varepsilon(\tau) \sinh |\tau|+\frac{\sqrt{\mu^{2}+1}}{\pi^{2}} \theta\left(d^{2}-\tau^{2}\right) \\
& \times \int_{0}^{\sqrt{d^{2}-\tau^{2}}} K_{1}\left(\sqrt{\mu^{2}+1} y\right) \sinh \sqrt{\tau^{2}+y^{2}} d y+\frac{\sqrt{\mu^{2}+1}}{2 \pi} \\
& \times\left(\theta\left(\tau^{2}-d^{2}\right) \int_{\sqrt{\tau^{2}-d^{2}}}^{|\tau|}+\theta\left(d^{2}-\tau^{2}\right) \int_{0}^{|\tau|}\right)\left(N_{1}\left(\sqrt{\mu^{2}+1} y\right)\right. \\
& \left.+i \varepsilon(\tau) J_{1}\left(\sqrt{\mu^{2}+1} y\right)\right) \sinh \sqrt{\tau^{2}-y^{2}} d y .
\end{aligned}
$$

In the $\theta\left(d^{2}-\tau^{2}\right)$-terms, there are singularities in $K_{1}$ and $N_{1}$ for $y \rightarrow 0$, cf. (3.23), which, however, cancel each other. As a consistency check, we note $\int_{0}^{d} \widetilde{S}_{1}^{T}(\tau, x) d x=\widetilde{S}_{0}^{T}(\tau, 0)-\widetilde{S}_{0}^{T}(\tau, d)$, which can readily be verified by $\left(J_{0}^{\prime}, N_{0}^{\prime}, K_{0}^{\prime}\right)(z)=-\left(J_{1}, N_{1}, K_{1}\right)(z)$. The integrals in (5.17) and (5.18) are of indefinite type and cannot be further evaluated in closed form, but this representation is sufficiently explicit to reveal the singularity and asymptotic structure and in particular the support of the propagators. The most surprising feature is perhaps, that the Feynman and Dyson propagators defined by (5.10) have an infrared singularity for $\mu \rightarrow 0$. Hence, for the electromagnetic field, these propagators are ill-defined by the standard contour integration, unless one uses some kind of infrared regularization such as a finite photon mass. This is an effect of the space curvature; if one approximates in (5.6) and (5.7), in the limit of large curvature radius, $\cosh d \sim 1$, and $\sinh d \sim d$, then the $\log \mu$ singularities stemming from $N_{0}$ and $K_{0}$ cancel each other. (Throughout the calculations, we have put the curvature radius of the 3 -space equal to one, but it can readily be restored by dimensional considerations, see below.) In retarded and advanced Green functions, cf. (5.9), the $\log \mu$-singularities cancel without performing this curvature limit. It would be interesting to investigate whether the infrared divergence of the quantum propagators can affect the emission probability of soft photons. ${ }^{16}$ If so, one could possibly draw inferences on the curvature sign.

Making use of (5.13), (5.14), (5.17), and (5.18), we readily find

$$
\begin{gathered}
S_{0}^{ \pm}(\tau, d ; \mu)=\theta( \pm \tau) \theta\left(\tau^{2}-d^{2}\right) J_{0}\left(\mu \sqrt{\tau^{2}-d^{2}}\right) \\
S_{1}^{ \pm}(\tau, d ; \mu)=2 d \theta( \pm \tau) \delta\left(d^{2}-\tau^{2}\right)-\mu d \theta( \pm \tau) \frac{\theta\left(\tau^{2}-d^{2}\right)}{\sqrt{\tau^{2}-d^{2}}} J_{1}\left(\mu \sqrt{\tau^{2}-d^{2}}\right) \\
\int_{0}^{d} S_{0}^{ \pm}(\tau, x ; \mu) d x=\theta( \pm \tau)\left(\theta\left(\tau^{2}-d^{2}\right) \int_{\sqrt{\tau^{2}-d^{2}}}^{|\tau|}+\theta\left(d^{2}-\tau^{2}\right) \int_{0}^{|\tau|}\right) J_{0}(\mu y) \frac{y d y}{\sqrt{\tau^{2}-y^{2}}}, \\
\int_{0}^{d} S_{1}^{ \pm}\left(\tau, x ; \sqrt{1+\mu^{2}}\right) \sinh x d x \\
=\theta( \pm \tau) \theta\left(d^{2}-\tau^{2}\right) \sinh |\tau|-\sqrt{\mu^{2}+1} \theta( \pm \tau) \\
\times\left(\theta\left(\tau^{2}-d^{2}\right) \int_{\sqrt{\tau^{2}-d^{2}}}^{|\tau|}+\theta\left(d^{2}-\tau^{2}\right) \int_{0}^{|\tau|}\right) J_{1}\left(\sqrt{\mu^{2}+1} y\right) \sinh \sqrt{\tau^{2}-y^{2}} d y
\end{gathered}
$$


and the $S_{i}^{ \pm}\left(\tau, d ; \sqrt{1+\mu^{2}}\right)$ are obtained by the substitution $\mu \rightarrow \sqrt{1+\mu^{2}}$. The retarded and advanced Green functions $G_{i j}^{ \pm}(\tau ; \mathbf{x}, \mathbf{y})$ of a massive vector field are thus given by (5.5)-(5.7), with (5.19) and (5.20) inserted. The analogous coefficients for the Feynman propagator read as

$$
\begin{gathered}
S_{0 F}^{+}(\tau, d ; \mu)=\frac{i}{\pi} \theta\left(d^{2}-\tau^{2}\right) K_{0}\left(\mu \sqrt{d^{2}-\tau^{2}}\right)+\frac{1}{2} \theta\left(\tau^{2}-d^{2}\right)\left(J_{0}\left(\mu \sqrt{\tau^{2}-d^{2}}\right)-i N_{0}\left(\mu \sqrt{\tau^{2}-d^{2}}\right)\right) \\
S_{1 F}^{+}(\tau, d ; \mu)=d \delta\left(d^{2}-\tau^{2}\right)+\frac{i \mu d}{\pi} \frac{\theta\left(d^{2}-\tau^{2}\right)}{\sqrt{d^{2}-\tau^{2}}} K_{1}\left(\mu \sqrt{d^{2}-\tau^{2}}\right) \\
-\frac{\mu d}{2} \frac{\theta\left(\tau^{2}-d^{2}\right)}{\sqrt{\tau^{2}-d^{2}}}\left(J_{1}\left(\mu \sqrt{\tau^{2}-d^{2}}\right)-i N_{1}\left(\mu \sqrt{\tau^{2}-d^{2}}\right)\right) \\
\begin{aligned}
\int_{0}^{d} S_{0 F}^{+}(\tau, x ; \mu) d x=\frac{i}{\pi} \theta\left(d^{2}-\tau^{2}\right) \int_{0}^{\sqrt{d^{2}-\tau^{2}}} \frac{K_{0}(\mu y) y d y}{\sqrt{\tau^{2}+y^{2}}+\frac{1}{2}}\left(\theta\left(\tau^{2}-d^{2}\right) \int_{\sqrt{\tau^{2}-d^{2}}}^{|\tau|}+\theta\left(d^{2}-\tau^{2}\right)\right. \\
\left.\times \int_{0}^{|\tau|}\right)\left(J_{0}(\mu y)-i N_{0}(\mu y)\right) \frac{y d y}{\sqrt{\tau^{2}-y^{2}}}, \\
\int_{0}^{d} S_{1 F}^{+}\left(\tau, x ; \sqrt{1+\mu^{2}}\right) \sinh x d x= \\
\frac{1}{2} \theta\left(d^{2}-\tau^{2}\right) \sinh |\tau|+\frac{i}{\pi} \sqrt{\mu^{2}+1} \theta\left(d^{2}-\tau^{2}\right) \\
\times \int_{0}^{\sqrt{d^{2}-\tau^{2}}} K_{1}\left(\sqrt{\mu^{2}+1} y\right) \sinh \sqrt{\tau^{2}+y^{2}} d y-\frac{1}{2} \sqrt{\mu^{2}+1} \\
\times\left(\theta\left(\tau^{2}-d^{2}\right) \int_{\sqrt{\tau^{2}-d^{2}}}^{|\tau|}+\theta\left(d^{2}-\tau^{2}\right) \int_{0}^{|\tau|}\right)\left(J_{1}\left(\sqrt{\mu^{2}+1} y\right)\right. \\
\left.-i N_{1}\left(\sqrt{\mu^{2}+1} y\right)\right) \sinh \sqrt{\tau^{2}-y^{2}} d y .
\end{aligned}
\end{gathered}
$$

The coefficients for the Dyson propagator are obtained by complex conjugation, $S_{i F}^{-}(\tau, d ; \mu)$ $=\overline{S_{i F}^{+}(\tau, d ; \mu)}$.

In the retarded and advanced propagators, the limit $\mu \rightarrow 0$ can be performed, and we find

$$
\begin{aligned}
G_{i j}^{ \pm}(\tau ; \mathbf{x}, \mathbf{y} ; \mu=0)= & -e^{-d / R}\left(R_{11}^{ \pm}(\tau, d ; \mu=0) \sinh (d / R) R d_{, i, j}\right. \\
& \left.+R_{33}^{ \pm}(\tau, d ; \mu=0) d_{, i} d_{, j}\right),
\end{aligned}
$$

with the matrix elements, cf. (5.19) and (5.20),

$$
\begin{aligned}
R_{11}^{ \pm}(\tau, d ; \mu=0)= & \frac{e^{d / R} \theta( \pm \tau)}{4 \pi R^{2} \sinh ^{3}(d / R)}\left[2 d R \sinh ^{2}\left(\frac{d}{R}\right) \delta\left(d^{2}-c^{2} \tau^{2}\right)+\theta\left(c^{2} \tau^{2}-d^{2}\right)\right. \\
& \times\left(-\frac{d}{R}+\sinh \frac{d}{R} \cosh \frac{d}{R}-\frac{1}{R} \int_{\sqrt{c^{2} \tau^{2}-d^{2}}}^{c|\tau|} J_{1}(y / R) \sinh \left(R^{-1} \sqrt{c^{2} \tau^{2}-y^{2}}\right) d y\right) \\
& +\theta\left(d^{2}-c^{2} \tau^{2}\right)\left(-\frac{c|\tau|}{R}+\sinh \frac{c|\tau|}{R}\right. \\
& \left.\left.-\frac{1}{R} \int_{0}^{c|\tau|} J_{1}(y / R) \sinh \left(R^{-1} \sqrt{c^{2} \tau^{2}-y^{2}}\right) d y\right)\right]
\end{aligned}
$$




$$
\begin{aligned}
R_{33}^{ \pm}(\tau, d ; \mu=0)= & \frac{e^{d / R} \theta( \pm \tau)}{2 \pi R^{2} \sinh ^{3}(d / R)}\left[d R \sinh ^{2}\left(\frac{d}{R}\right) \delta\left(d^{2}-c^{2} \tau^{2}\right)+\theta\left(c^{2} \tau^{2}-d^{2}\right)\right. \\
& \times\left(\frac{d}{R} \cosh \frac{d}{R}-\sinh \frac{d}{R}-\frac{d}{2} \sinh ^{2}\left(\frac{d}{R}\right) \frac{J_{1}\left(R^{-1} \sqrt{c^{2} \tau^{2}-d^{2}}\right)}{\sqrt{c^{2} \tau^{2}-d^{2}}}\right. \\
& \left.+\cosh \left(\frac{d}{R}\right) \frac{1}{R} \int_{\sqrt{c^{2} \tau^{2}-d^{2}}}^{c|\tau|} J_{1}(y / R) \sinh \left(R^{-1} \sqrt{c^{2} \tau^{2}-y^{2}}\right) d y\right)+\theta\left(d^{2}-c^{2} \tau^{2}\right) \\
& \left.\times \cosh \frac{d}{R}\left(\frac{c|\tau|}{R}-\sinh \frac{c|\tau|}{R}+\frac{1}{R} \int_{0}^{c|\tau|} J_{1}(y / R) \sinh \left(R^{-1} \sqrt{c^{2} \tau^{2}-y^{2}}\right) d y\right)\right] .
\end{aligned}
$$

We have here restored the natural units, the curvature radius $R$ of the 3 -space, and $c$. [The exponentials drop out, of course, we just have them included to be consistent with the previous notation, and they are also convenient when considering the $d \rightarrow \infty$ asymptotics, see after (6.14).] In the $B^{3}$-model, we recover for $R \rightarrow \infty$ the Green functions in Minkowski space, cf. the discussion preceding (3.31). In fact, $d d_{, i, j}+d_{, i} d_{, j} \sim-4 \delta_{i j}$, so that (5.23) boils down to

$$
G_{i j}^{ \pm}(\tau ; \mathbf{x}, \mathbf{y} ; \mu=0, R \rightarrow \infty) \sim \frac{1}{2 \pi} 4 \delta_{i j} \theta( \pm \tau) \delta\left(4|\mathbf{x}-\mathbf{y}|^{2}-c^{2} \tau^{2}\right),
$$

supported only at the light cone. By a rescaling of the space coordinates, one can get rid of the two factors of 4 . One can sometimes switch from negative to positive curvature just by analtyic continuation in the curvature radius, and it would be interesting to check in how far this persists here.

Up to now we considered a static RW cosmology [expansion factor $a(\tau)=1$ ]; the retarded and advanced electromagnetic Green functions as defined by (3.30) and (5.23) remain valid, with minor modifications, in a general RW cosmology, $d s^{2}=-d \tau^{2}+a^{2}(\tau) d \sigma^{2}$. In the spectral elementary waves (2.15) and (2.16), we have to substitute $\tau \rightarrow \int_{\tau_{0}}^{\tau} a^{-1}(\tau) d \tau$ into the exponentials, so that the frequencies of the transversal and longitudinal waves scale as $|s| a^{-1}(\tau)$ and $\sqrt{s^{2}+1} a^{-1}(\tau)$, respectively. In addition, the longitudinal time component $A_{0}^{L}$ in (2.16) has to be rescaled by a factor $a^{-1}(\tau)$. In the Green functions (3.30) and (5.23), we have to perform the same substitution for the time variable, and then to scale $G_{00}^{ \pm}$by a factor $\left(a(\tau) a\left(\tau_{0}\right)\right)^{-1}$. These modifications only apply to the massless case, $\mu=0$, as a consequence of the conformal coupling of the electromagnetic field. If the rest mass is finite, a time separation of (2.4)-(2.6) is still possible, so that the time independent part of the spectral problem, in particular the resolvent kernel calculated in Secs. III and IV, also applies to a RW cosmology with arbitrary expansion factor without modifications. However, the time dependence of the eigenfunctions is then not any more exponential as in (2.15) and (2.16), and has to be calculated from scratch, and the integral transform (2.28) relating the time dependent Green functions to the resolvent kernel must be modified accordingly. In a Milne universe with linear expansion factor, $a(\tau)=(c / R) \tau$, it should be possible to obtain quite explicit formulas for the massive propagators. ${ }^{17}$

Remark: The time component $G_{00(F)}^{ \pm}\left(\mu^{2}=-1\right)$, if scaled in the indicated way with the expansion factor, also defines the propagators of the conformally coupled Klein-Gordon equation, as discussed at the end of Sec. III. The limit $\mu^{2} \rightarrow-1$ can easily be carried out in (3.25) and (3.26). By a standard scaling argument ${ }^{2}$ applied to (3.32), we find

$$
(\square-\hat{R} / 6) G_{00(F)}^{ \pm}\left(\tau, d ; \mu^{2}=-1\right)=a^{-3}(\tau) \delta_{B^{3}}(\mathbf{x}, \mathbf{y}) \delta(\tau),
$$

where $\square$ is the d'Alembertian of the RW-line element, and 


$$
\hat{R}:=6\left(\frac{-1}{a^{2}}+\frac{\ddot{a}}{a}+\frac{\dot{a}^{2}}{a^{2}}\right)
$$

is the Ricci scalar. The spectral elementary waves, solving $(\square-\hat{R} / 6) \psi=0$, read

$$
\psi=a^{-1}(\tau) \exp \left( \pm i s \int a^{-1}(\tau) d \tau\right) P^{1+i s}
$$

with the Poisson kernel $P$ as defined in (2.17).

\section{GREEN FUNCTIONS IN RW COSMOLOGIES WITH MULTIPLY CONNECTED HYPERBOLIC 3-SPACE}

We start with the time independent Green functions $\hat{G}_{00}(\omega ; \mathbf{x}, \mathbf{y})$, cf. (3.6) and $\hat{G}_{i j}(\omega ; \mathbf{x}, \mathbf{y})$, cf. (4.25). For $\lambda$, we substitute $\lambda_{(F)}^{ \pm}(\omega)$ as in (3.16) and write accordingly, in the $B^{3}$-model, $\hat{G}_{(F) 00}^{ \pm}(\omega ; \mathbf{x}, \mathbf{y})$ and $\hat{G}_{(F) i j}^{ \pm}(\omega ; \mathbf{x}, \mathbf{y})$, as at the beginning of Sec. V. We will switch between the $B^{3}$ and $H^{3}$-models whenever convenient; in the $H^{3}$-model, we write $\hat{G}_{(F) \alpha \beta}^{ \pm}\left(\omega ; z, t ; z_{0}, t_{0}\right)$. Green functions on multiply connected hyperbolic manifolds can formally be obtained by periodizing $B^{3}$ or $H^{3}$-Green functions with the covering group $\Gamma$,

$$
\begin{gathered}
\hat{G}_{(F) 00}^{\Gamma \pm}(\omega ; \mathbf{x}, \mathbf{y}):=\sum_{\gamma \in \Gamma} \hat{G}_{(F) 00}^{ \pm}(\omega ; \gamma \mathbf{x}, \mathbf{y}), \\
\hat{G}_{(F) i j}^{\Gamma \pm}(\omega ; \mathbf{x}, \mathbf{y}):=\sum_{\gamma \in \Gamma} \hat{G}_{(F) k j}^{ \pm}(\omega ; \gamma \mathbf{x}, \mathbf{y})\left[\gamma^{\prime} \mathbf{x}\right]_{k i} ;
\end{gathered}
$$

the scalar case (6.1) is well documented. ${ }^{6,9,10,18}$ The Jacobian $\left[\gamma^{\prime} \mathbf{x}\right]$ in (6.2) is readily calculated by making use of the representation ${ }^{5} \gamma(\mathbf{x})=k T_{\gamma^{-1}(\mathbf{0})} \mathbf{x}$, with a constant matrix $k \in \mathrm{SO}(3)$; the Jacobian of $T_{\mathbf{y}} \mathbf{x}$ is given in (4.15).

The hard part is of course to show that the series (6.1) and (6.2) converge, see after (6.11). We restrict this investigation to hyperbolic manifolds that admit a finitely sided fundamental polyhedron without parabolic cusp singularities. The covering group $\Gamma$ is a Kleinian group ${ }^{19-24}$ a discrete subgroup of $\operatorname{SL}(2, C) /\{ \pm i d\}$ acting via 3D Möbius transformations in $B^{3}$ or $H^{3}$. $\Gamma$ is finitely generated, and does not contain parabolic (or elliptic) elements. As for the Hausdorff dimension $\delta$ of the limit set of $\Gamma$, the manifold is open if $0 \leqslant \delta<2$, so that the fundamental polyhedron has faces on the boundary of $H^{3}$; it is compact if $\delta=2$, in this case the fundamental polyhedron does not touch the boundary. The domain defined by the polyhedral faces on the boundary is denoted by $f$; it is a fundamental domain of $\Gamma$ acting via Möbius (i.e., linear fractional) transformations in the complex plane. Examples for open hyperbolic manifolds are solid handle-bodies with Schottky covering groups; ${ }^{19}$ the limit sets of these groups are Cantor sets with $0<\delta<1$. Another class of open hyperbolic manifolds are thickened Riemann surfaces, hollow handle bodies, with (quasi-) Fuchsian ${ }^{20}$ covering groups, whose limit sets are closed Jordan curves with Hausdorff dimensions $1 \leqslant \delta<2$. As mentioned, the faces of the fundamental polyhedron corresponding to the surfaces of these 3D handle bodies lie on the boundary at infinity of hyperbolic space, i.e., in the complex plane, if we use the $H^{3}$-model, and so these manifolds are open. In the following only manifolds with $0 \leqslant \delta<1$ are considered, to assure the convergence of the series (6.1) and (6.2).

The periodization in (6.1) and (6.2) may as well be with respect to $\mathbf{y}$, because $\hat{G}_{(F) 00}^{ \pm}$and $\hat{G}_{(F) i j}^{ \pm}$are scalar and vectorial point-pair invariants, respectively,

$$
\hat{G}_{(F) 00}^{ \pm}(\omega ; \gamma \mathbf{x}, \gamma \mathbf{y})=\hat{G}_{(F) 00}^{ \pm}(\omega ; \mathbf{x}, \mathbf{y}),
$$




$$
\begin{gathered}
\hat{G}_{(F) i j}^{ \pm}(\omega ; \gamma \mathbf{x}, \gamma \mathbf{y})\left[\gamma^{\prime} \mathbf{x}\right]_{i m}\left[\gamma^{\prime} \mathbf{y}\right]_{j n}=\hat{G}_{(F) m n}^{ \pm}(\omega ; \mathbf{x}, \mathbf{y}), \\
\hat{G}_{(F) k n}^{ \pm}(\omega ; \gamma \mathbf{x}, \mathbf{y})\left[\gamma^{\prime} \mathbf{x}\right]_{k m}=\hat{G}_{(F) m k}^{ \pm}\left(\omega ; \mathbf{x}, \gamma^{-1} \mathbf{y}\right)\left[\gamma^{-1,} \mathbf{y}\right]_{k n} .
\end{gathered}
$$

These symmetries hold for every Möbius transformation in $B^{3}$, unrelated to the covering group $\Gamma$, because $\hat{G}_{(F) 00}^{ \pm}$and $\hat{G}_{(F) i j}^{ \pm}$are functions of the scalar point-pair invariant $L(\mathbf{x}, \mathbf{y})$, and the vectorial invariants $\partial L / \partial x_{i} \partial L / \partial y_{j}$ and $\partial^{2} L / \partial x_{i} \partial y_{j}$. The periodization (6.1) and (6.2) may be replaced by a $\Gamma$-periodization with respect to $\mathbf{y}$ (and $j$ ). To see this, we replace in (6.3) and (6.4) $\mathbf{x}$ by $\gamma^{-1} \mathbf{x}$, and because the summation in (6.1) and (6.2) is over the whole group, we may replace there $\gamma$ by $\gamma^{-1}$. By a similar reasoning, (6.3) and (6.4) still holds for the periodized functions (6.1) and (6.2), provided $\gamma \in \Gamma$.

The spectral representation of $\hat{G}_{(F) \alpha \beta}^{\Gamma \pm}$ is readily derived in the half-space model $H^{3}$. Starting with the scalar time component, we define the Eisenstein series ${ }^{6,9,10}$

$$
E(z, t ; \xi, 1+i s):=\sum_{\gamma \in \Gamma} P^{1+i s}(\gamma(z, t) ; \xi)=\sum_{\gamma \in \Gamma}\left|\gamma^{\prime} \xi\right|^{1+i s} P^{1+i s}(z, t ; \gamma \xi),
$$

where we used the symmetry (2.24), which also implies

$$
E(\gamma(z, t) ; \xi, 1+i s)=E(z, t ; \xi, 1+i s), \quad\left|\gamma^{\prime} \xi\right|^{1+i s} E(z, t ; \gamma \xi, 1+i s)=E(z, t ; \xi, 1+i s),
$$

for $\gamma \in \Gamma$. In the first equation in (2.16), we substitute the series (6.5) for $P^{1+i s}$, which amounts to periodizing the $H^{3}$-eigenfunctions with respect to the hyperbolic lattice. The series (6.5) converges for the indicated covering groups (with $\delta<1$ ), see after (6.11). It is easy to see, that the orthogonality and completeness relations (A12) and (A14) remain valid for $E(z, t ; \xi, 1 \pm i s)$ with some obvious modifications; apart from replacing $P^{1 \pm i s}$ by $E(z, t ; \xi, 1 \pm i s)$, the domain of integration $\left(H^{3}\right)$ in (A12) has to be restricted to a fundamental polyhedron $F$ of the covering group, and in (A14) the domain of integration $\left(R^{2} \times R^{+}\right)$is restricted to $f \times R^{+}$, where $f$ is a fundamental domain of $\Gamma$ in the complex plane. [The formal procedures to show this, and to derive (6.7) and (6.10) below, are quite similar to those indicated in (6.24) and (6.25).] It should be stressed, that these statements are only safe for the specified covering groups. For example, the series (6.5) diverges for covering groups as defined after (6.2) with $\delta \geqslant 1$, and analytic continuation is needed to define it for real $s$. This is not only a technicality, because bound states emerge in addition to the continuous spectrum, ${ }^{6}$ and the set $(6.5)$ is not complete.

If $\delta<1$, the completeness relation (A14) holds with the indicated modifications, and we may write, analogously to (3.13),

$$
\hat{G}_{(F) 00}^{\Gamma \pm}\left(\omega ; z, t ; z_{0}, t_{0}\right)=-\int_{f \times R^{+}} d \sigma(\xi, s) \frac{E(z, t ; \xi, 1+i s) E\left(z_{0}, t_{0} ; \xi, 1-i s\right)}{s^{2}+1+\lambda_{(F)}^{ \pm}(\omega)},
$$

with the spectral measure defined in (A13). The formal identity of (6.7) with (6.1) can be easily shown by means of the completeness relation and the spectral representation (3.13) in the covering space, along the lines of (6.24) and (6.25).

The spatial component of the resolvent kernel admits a similar representation. The vectorial analog to $(6.5)$ reads as ${ }^{13}$

$$
\begin{gathered}
\mathbf{a}_{j}^{\Gamma T_{L}}(z, t ; \xi, s):=\sum_{\gamma \in \Gamma} \mathbf{a}_{i}^{T_{L}}(\gamma(z, t) ; \xi, s)\left[\gamma^{\prime}(z, t)\right]_{i j}=\sum_{\gamma \in \Gamma}\left|\gamma^{\prime} \xi\right|^{i s} \overline{\gamma^{\prime} \xi} \mathbf{a}_{j}^{T_{L}}(z, t ; \gamma \xi, s), \\
\mathbf{a}_{j}^{\Gamma T_{R}}(z, t ; \xi, s):=\sum_{\gamma \in \Gamma}\left|\gamma^{\prime} \xi\right|^{i s} \gamma^{\prime} \xi \mathbf{a}_{j}^{T_{R}}(z, t ; \gamma \xi, s), \\
\mathbf{a}_{j}^{\Gamma L}(z, t ; \xi, s):=\sum_{\gamma \in \Gamma}\left|\gamma^{\prime} \xi\right|^{1+i s} \mathbf{a}_{j}^{L}(z, t ; \gamma \xi, s),
\end{gathered}
$$


with the invariance property, cf. (6.6),

$$
\mathbf{a}_{i}^{\Gamma T_{L}}(\gamma(z, t) ; \xi, s)\left[\gamma^{\prime}(z, t)\right]_{i j}=\mathbf{a}_{j}^{\Gamma T_{L}}(z, t ; \xi, s)=\left|\gamma^{\prime} \xi\right|^{i s} \bar{\gamma}^{\prime} \xi \mathbf{a}_{j}^{\Gamma T_{L}}(z, t ; \gamma \xi, s),
$$

and analogous relations hold for the right circular and longitudinal polarizations. These Eisenstein series are obtained by periodizing the circularly polarized transversal vectors $\mathbf{a}_{j}^{T_{L, R}}$ as defined in (2.22), and the longitudinal vector $\mathbf{a}_{j}^{L}$ in (2.20). The symmetries (2.23) have been invoked in (6.8) and (6.9). Again, for the indicated covering groups (with $\delta<1$ ), these series converge for real $s$, see the discussion following (6.11). The spatial components of the eigenfunctions in (2.15) and (2.16) get periodized (and circularly polarized) by replacing there $\mathbf{a}_{j}^{T_{k}, L}$ by (6.8), and then they solve again the $\Gamma$-invariant Eq. (2.6). The vectors in (6.8) are orthogonal, cf. (B4)-(B6), and the completeness relation (B14) stays valid with the substitutions $\mathbf{a}^{T_{k}, L} \rightarrow \mathbf{a}^{\Gamma T_{L, R}, L}$ and the abovementioned restriction of the domain of integration to a fundamental domain. Accordingly, the spectral resolution of the matrix elements (4.1) and (4.2) reads

$$
\begin{gathered}
\hat{R}_{i j}^{\Gamma T}\left(z, t ; z_{0}, t_{0} ; \lambda\right):=\int_{f \times R^{+}} \sum_{k=L, R} \mathbf{a}_{i}^{\Gamma T_{k}}(z, t ; \xi, s) \overline{\mathbf{a}_{j}^{\Gamma T_{k}}\left(z_{0}, t_{0} ; \xi, s\right)} \frac{d \sigma^{T}(\xi, s)}{s^{2}+\lambda}, \\
\hat{R}_{i j}^{\Gamma L}\left(z, t ; z_{0}, t_{0} ; \lambda\right):=\int_{f \times R^{+}} \mathbf{a}_{i}^{\Gamma L}(z, t ; \xi, s) \overline{\mathbf{a}_{j}^{\Gamma L}\left(z_{0}, t_{0} ; \xi, s\right)} \frac{d \sigma^{L}(\xi, s)}{s^{2}+1+\lambda},
\end{gathered}
$$

and the spectral representation of the periodized spatial Green function is thus found as

$$
\hat{G}_{(F) i j}^{\Gamma \pm}\left(\omega ; z, t ; z_{0}, t_{0}\right)=\hat{R}_{i j}^{\Gamma T}\left(z, t ; z_{0}, t_{0} ; \lambda_{(F)}^{ \pm}(\omega)\right)+\hat{R}_{i j}^{\Gamma L}\left(z, t ; z_{0}, t_{0} ; \lambda_{(F)}^{ \pm}(\omega)\right) .
$$

[The $k$-summation in $\hat{R}_{i j}^{\Gamma T}$ is over left and right circular polarizations, which may as well be replaced by linearly polarized states, according to (2.22).] The spectral measures in (6.10) remain as defined in (B13), but the domain of the $\xi$-integration is restricted to a fundamental domain $f$ in the complex plane.

The Poisson kernel $P(z, t ; \gamma \xi)$ as well as the components of the vectors $\mathbf{a}^{T_{L, R}, L}(z, t ; \gamma \xi, s)$ are bounded for $\gamma \in \Gamma$, and hence the convergence abscissa of the Eisenstein series (6.5) and (6.8) coincides with that of the Poincare series $\Sigma_{\gamma \in \Gamma}\left|\gamma^{\prime} \xi\right|^{1+i s}$. (The limit set is the set of accumulation points of the orbit $\gamma \xi$, by the way.) As for the covering groups indicated after (6.2), this series is known to converge only for $\operatorname{Re}(1+i s)>\delta$, cf. Ref. 6 .

To obtain the convergence behavior of the Poincare series (6.1) and (6.2), we need an estimate on $\hat{G}_{(F) \alpha \beta}^{ \pm}(\omega ; \mathbf{x}, \mathbf{y})$ [defined by (3.6) and (4.25)] for $d \rightarrow \infty$, which means for $|\mathbf{x}| \rightarrow 1$, and $\mathbf{y}$ fixed, or vice versa. For the time component, this is easily settled, $\hat{G}_{00}(\omega ; \mathbf{x}, \mathbf{y})=O(\exp (-(1+\widetilde{\varepsilon}) d(\mathbf{x}, \mathbf{y})))$, with $\widetilde{\varepsilon}:=\operatorname{Re} \sqrt{1+\lambda}$, cf. (3.6). When calculating the real space Green functions (3.15), the limit $\widetilde{\varepsilon} \rightarrow 0$ is attained.

Next we turn to the $d \rightarrow \infty$ asymptotics of the space component and the Jacobian in (6.2). In the $B^{3}$-model, we can readily calculate $(\log L)_{, i, j}$, cf. (3.5), in the limit $|\mathbf{x}| \rightarrow 1$,

$$
\frac{L_{, i, j}}{L}-\frac{L_{, i} L_{, j}}{L^{2}} \sim \frac{-4}{1-2 \mathbf{x y}+\mathbf{y}^{2}}\left(\frac{1}{2} \delta_{i j}-\frac{\left(x_{i}-y_{i}\right)\left(x_{j}-y_{j}\right)}{1-2 \mathbf{x y}+\mathbf{y}^{2}}\right) .
$$

[As in (4.21), $i$ means differentiation with respect to $x_{i}$, and $j$ with respect to $y_{j}$.] Likewise, for $|\mathbf{x}| \rightarrow 1$,

$$
\frac{L_{, i} L_{, j}}{L^{2}} \sim \frac{4 x_{i}\left(2 y_{j}(1-\mathbf{x y})-x_{j}\left(1-\mathbf{y}^{2}\right)\right)}{\left(1-\mathbf{x}^{2}\right)\left(1-\mathbf{y}^{2}\right)\left(1-2 \mathbf{x y}+\mathbf{y}^{2}\right)} .
$$

Moreover, we find from (4.24), for $L \rightarrow \infty$, 


$$
\begin{gathered}
d_{, i} d_{, j} \sim L_{, i} L_{, j} / L^{2}=O(L), \\
\sinh (d) d_{, i, j} \sim 2 L_{, i, j}-2 \frac{L_{, i} L_{, j}}{L}+\frac{L_{, i} L_{, j}}{L^{2}}=O(L),
\end{gathered}
$$

Clearly, $L \sim e^{d} / 4$, so that $e^{-d} d_{, i} d_{, j}=O(1)$, as well as $e^{-d} \sinh (d) d_{, i, j}=O(1)$, for $d \rightarrow \infty$. Accordingly, $\hat{R}_{11}=O\left(e^{-\sqrt{\lambda} d}\right)$, and $\hat{R}_{33}=O\left(e^{-\sqrt{1+\lambda} d}\right)$, cf. (4.26) and (4.27), and hence the asymptotics of the Green function (4.25) is evident, $\quad \hat{G}_{i j}(\omega ; \mathbf{x}, \mathbf{y})=O(\exp (-\widetilde{\varepsilon} d(\mathbf{x}, \mathbf{y})))$, with $\widetilde{\varepsilon}$ $:=\min (\operatorname{Re} \sqrt{\lambda}, \operatorname{Re} \sqrt{1+\lambda})>0$. In the integration procedures for the time dependent Green functions, cf. (5.4) and (3.16), the limit $\widetilde{\varepsilon} \rightarrow 0$ is again reached. In (6.2), the estimate for $\hat{G}_{i j}$ has still to be multiplied with the estimate for the Jacobian $\left[\gamma^{\prime} \mathbf{x}\right]$.

As for the Jacobian $\hat{x}_{j, i}$ of $T_{\mathbf{y}} \mathbf{x}$, cf. (4.15), we find, for fixed $\mathbf{x}$ and $|\mathbf{y}| \rightarrow 1, \hat{x}_{j, i}=O(1-|\mathbf{y}|)$.

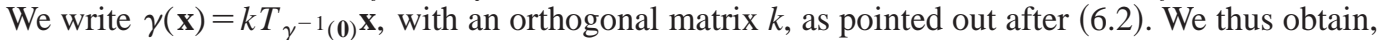
for $\left|\gamma^{-1}(\mathbf{0})\right| \rightarrow 1$, the estimate $\left[\gamma^{\prime} \mathbf{x}\right]_{m n}=O\left(1-\left|\gamma^{-1} \mathbf{0}\right|\right)$. Using $L(\mathbf{0}, \gamma(\mathbf{y}))=L\left(\gamma^{-1}(\mathbf{0}), \mathbf{y}\right)$, we find $\left[\gamma^{\prime} \mathbf{x}\right]_{m n}=O(1-|\gamma \mathbf{y}|)$, for $|\gamma \mathbf{y}| \rightarrow 1$, with fixed $\mathbf{x}$ and $\mathbf{y}$, for every Kleinian group, and so we may write $\left[\gamma^{\prime} \mathbf{x}\right]_{m n}=O(\exp (-d(\mathbf{y}, \gamma \mathbf{x})))=O(\exp (-d(\gamma \mathbf{y}, \mathbf{x})))$. (The geometric reasoning underlying such estimates, also with regard to uniformity in fundamental domains, is explained in Ref. 5.) We conclude, that the convergence of the series (6.1) and (6.2) is decided by the convergence of $\Sigma_{\gamma \in \Gamma} \exp (-d(\gamma \mathbf{x}, \mathbf{y}))$, uniformly in $\lambda=\lambda_{(F)}^{ \pm}(\varepsilon, \omega)$, for real $\omega$ and $\varepsilon \rightarrow 0$. The Poincaré series $\Sigma_{\gamma \in \Gamma} \exp (-\alpha d(\gamma \mathbf{x}, \mathbf{y}))$ and $\Sigma_{\gamma \in \Gamma}\left|\gamma^{\prime} \xi\right|^{\alpha}$ have the same abscissa of convergence. Accordingly, in the case of the covering groups specified after (6.2), we can use the series (6.1) and (6.2) only if $\delta<1$, which also happens to be the criterion for the convergence of the Eisenstein series (6.5) and (6.8).

The time-dependent Green function on the 4-manifold is defined as the Fourier transform of (6.1) and (6.2), cf. (2.28), (3.15), and (5.4),

$$
G_{(F) \alpha \beta}^{\Gamma \pm}(\tau ; \mathbf{x}, \mathbf{y})=(2 \pi)^{-1} \int_{-\infty}^{+\infty} d \omega e^{-i \omega \tau} \hat{G}_{(F) \alpha \beta}^{\Gamma \pm}(\omega ; \mathbf{x}, \mathbf{y}) .
$$

By formally interchanging summation and integration, we find

$$
\begin{gathered}
G_{(F) 00}^{\Gamma \pm}(\tau ; \mathbf{x}, \mathbf{y})=\sum_{\gamma \in \Gamma} G_{(F) 00}^{ \pm}(\tau ; \gamma \mathbf{x}, \mathbf{y}), \\
G_{(F) i j}^{\Gamma \pm}(\tau ; \mathbf{x}, \mathbf{y})=\sum_{\gamma \in \Gamma} G_{(F) k j}^{ \pm}(\tau ; \gamma \mathbf{x}, \mathbf{y})\left[\gamma^{\prime} \mathbf{x}\right]_{k i},
\end{gathered}
$$

which means periodization of the real space Green functions defined in (3.28), (3.29), and (5.5)(5.7). The convergence of the series (6.16) and (6.17) can be estimated as above, $G_{(F) 00}^{ \pm}(\tau ; \mathbf{x}, \mathbf{y})$ $=O\left(e^{-d}\right)$, uniformly in $\tau$, for $|\mathbf{x}| \rightarrow 1$ and fixed $\mathbf{y}$, which follows from (3.28), (3.29), the asymptotics of the Bessel functions there, and (A10). Likewise, $G_{(F) i j}^{ \pm}(\tau ; \mathbf{x}, \mathbf{y})=O(1)$, which follows from (5.5)-(5.7) and (5.19)-(5.22). The Jacobian in (6.17) is $O\left(e^{-d}\right)$, so that we end up, like above, with $\Sigma_{\gamma \in \Gamma} e^{-d}$, as series determining the convergence of (6.16) and (6.17). Hence, for geometrically finite covering groups, without parabolic and elliptic elements, and with $\delta<1$, the propagators on the hyperbolic manifold are obtained by periodizing the corresponding Green functions in the covering space. Clearly, the symmetry (6.3) and (6.4) also applies to $G_{(F) \alpha \beta}^{\Gamma \pm}(\tau ; \mathbf{x}, \mathbf{y})$, for $\gamma \in \Gamma$.

Finally we point out a method to obtain wave solutions on the multiply connected manifold, that does not make explicit use of the periodized Green functions. This is not only useful for $\delta$ $\geqslant 1$, when the above series fail to converge, but also if $\delta<1$, because the series $\Sigma_{\gamma \in \Gamma}\left|\gamma^{\prime} \xi\right|^{\alpha}$ is not known for its rapid convergence. ${ }^{21}$ Wave fields satisfying the field Eqs. (2.1) on the multiply connected 4-manifold are obtained as 


$$
A_{\alpha}^{\Gamma}(\tau, \mathbf{x})=\int_{F \times R} G_{\alpha \alpha^{\prime}}^{\Gamma}\left(\tau-\tau^{\prime} ; \mathbf{x}, \mathbf{x}^{\prime}\right) j^{\Gamma \alpha^{\prime}}\left(\tau^{\prime}, \mathbf{x}^{\prime}\right) d V_{B^{3}} d \tau^{\prime}
$$

analogously to (2.26). The spatial integration is over a fundamental polyhedron $F$, and $G_{\alpha \alpha^{\prime}}^{\Gamma}$ denotes a linear combination of the propagators $G_{(F) \alpha \beta}^{\Gamma \pm}$, with coefficients adding up to unity, so that $G_{\alpha \alpha^{\prime}}^{\Gamma}$ solves (2.25). The current is periodic with respect to the covering group,

$$
j^{\Gamma 0}(\tau, \gamma \mathbf{x})=j^{\Gamma 0}(\tau, \mathbf{x}),\left[\gamma^{\prime} \mathbf{x}\right]^{-1 i k} j^{\Gamma k}(\tau, \gamma \mathbf{x})=j^{\Gamma i}(\tau, \mathbf{x}),
$$

and so is the resulting vector potential,

$$
A_{0}^{\Gamma}(\tau, \gamma \mathbf{x})=A_{0}^{\Gamma}(\tau, \mathbf{x}), A_{k}^{\Gamma}(\tau, \gamma \mathbf{x})\left[\gamma^{\prime} \mathbf{x}\right]_{k i}=A_{i}^{\Gamma}(\tau, \mathbf{x}),
$$

as $G_{\alpha \alpha^{\prime}}^{\Gamma}$ admits the symmetries stated in (6.3) and (6.4) for $\gamma \in \Gamma$. The current density $j^{\Gamma \alpha}(\tau, \mathbf{x})$ is usually obtained by periodizing a density $j^{\alpha}(\tau, \mathbf{x})$ in $B^{3}$ or $H^{3}$,

$$
j^{\Gamma 0}(\tau, \mathbf{x})=\sum_{\gamma \in \Gamma} j^{0}(\tau, \gamma \mathbf{x}), j^{\Gamma i}(\tau, \mathbf{x})=\sum_{\gamma \in \Gamma}\left[\gamma^{\prime} \mathbf{x}\right]^{-1 i k} j^{k}(\tau, \gamma \mathbf{x}) .
$$

Alternatively to (6.18), we may periodize the wave field

$$
A_{\alpha}(\tau, \mathbf{x})=\int_{B^{3} \times R} G_{\alpha \alpha^{\prime}}\left(\tau-\tau^{\prime} ; \mathbf{x}, \mathbf{x}^{\prime}\right) j^{\alpha^{\prime}}\left(\tau^{\prime}, \mathbf{x}^{\prime}\right) d V_{B^{3}} d \tau^{\prime},
$$

where $G_{\alpha \alpha^{\prime}}$ is again a linear combination of the propagators $G_{(F) \alpha \beta}^{ \pm}$in the covering space, so that

$$
A_{0}^{\Gamma}(\tau, \mathbf{x})=\sum_{\gamma \in \Gamma} A_{0}(\tau, \gamma \mathbf{x}), \quad A_{i}^{\Gamma}(\tau, \mathbf{x})=\sum_{\gamma \in \Gamma} A_{k}(\tau, \gamma \mathbf{x})\left[\gamma^{\prime} \mathbf{x}\right]_{k i} .
$$

This periodization is formally equivalent to (6.18), by way of (6.21), (6.16), and (6.17),

$$
\begin{aligned}
& \sum_{\gamma \in \Gamma} A_{0}(\tau, \gamma \mathbf{x})=\sum_{\gamma \in \Gamma} \sum_{\beta \in \Gamma} \int_{F \times R} G_{00}\left(\tau-\tau^{\prime} ; \beta^{-1} \gamma \mathbf{x}, \mathbf{x}^{\prime}\right) j^{0}\left(\tau^{\prime}, \beta \mathbf{x}^{\prime}\right) d V_{B^{3}}\left(\mathbf{x}^{\prime}\right) d \tau^{\prime} \\
& \sum_{\gamma \in \Gamma} A_{k}(\tau, \gamma \mathbf{x})\left[\gamma^{\prime} \mathbf{x}\right]_{k i}= \sum_{\gamma \in \Gamma} \sum_{\beta \in \Gamma} \int_{F \times R} G_{a b}\left(\tau-\tau^{\prime} ; \beta^{-1} \gamma \mathbf{x}, \mathbf{x}^{\prime}\right) \\
& \times\left[\beta^{-1 \prime}(\gamma \mathbf{x})\right]_{a k}\left[\gamma^{\prime} \mathbf{x}\right]_{k i}\left[\beta^{-1 \prime}\left(\beta \mathbf{x}^{\prime}\right)\right]_{b m} j^{m}\left(\tau^{\prime}, \beta \mathbf{x}^{\prime}\right) d V_{B^{3}}\left(\mathbf{x}^{\prime}\right) d \tau^{\prime}
\end{aligned}
$$

Here we at first used $B^{3}=\cup_{\beta \in \Gamma} \beta(F)$, and then the invariance of the hyperbolic volume element, $d V_{B^{3}}(\beta \mathbf{x})=d V_{B^{3}}(\mathbf{x})$, as well as the point-pair invariance (6.3) and (6.4). The first two Jacobians in (6.25) can be replaced by $\left[\left(\beta^{-1} \gamma\right)^{\prime} \mathbf{x}^{\prime}\right]_{a i}$, and the third by $\left[\beta^{\prime} \mathbf{x}^{\prime}\right]^{-1 b m}$. (As for Jacobians in Poincaré series, we do not distinguish between sub- and superscripts and avoid mixed indices; the summation convention applies whenever two indices are equal.) Next we pull the $\gamma \in \Gamma$ summation under the integral sign. As this summation is over the whole group $\Gamma$, we may drop the $\beta^{-1}$ in $G_{\alpha \beta}$ and in the Jacobian, and obtain in this way $G_{\alpha \alpha^{\prime}}^{\Gamma}$. The periodized current $j^{\Gamma \alpha}$ is finally recovered by pulling the $\beta \in \Gamma$ summation under the integral sign. In (6.23), no explicit use is made of the Green functions (6.16) and (6.17) on the 4-manifold.

\section{ACKNOWLEDGMENTS}

The author acknowledges the support of the Japan Society for the Promotion of Science. I would like to thank C. V. Vishveshwara for initiating my visit to the Indian Institute of Astrophys- 
ics, Bangalore. The hospitality and stimulating atmosphere of the Center for Nonlinear Dynamics, Bharathidasan University, Trichy, the Institute of Mathematical Sciences, Madras, and the Tata Institute of Fundamental Research, Bombay, are likewise gratefully acknowledged.

\section{APPENDIX A: ORTHOGONALITY, COMPLETENESS, AND THE RESOLVENT KERNEL FOR THE LONGITUDINAL TIME COMPONENT}

We calculate the resolvent kernel (3.14), and the spectral measure for the time component of the eigenvectors (2.16). The results obtained in this appendix are not new, and a more detailed account on the spectral theory of the hyperbolic Laplace-Beltrami operator, without explicit use of distributions, can be found in Refs. 9 and 10. I include this appendix, because the scalar Green function happens to be the time component of the electromagnetic propagators, and the integral (A1) is also a prerequisite for the calculation of the spatial resolvent kernel in Appendix B.

The key convolution of $H^{3}$-spectral theory is

$$
C_{\alpha, \beta}\left(z_{1}, t_{1} ; z_{2}, t_{2}\right)=\int_{R^{2}}\left(\left|z_{1}-\xi\right|^{2}+t_{1}^{2}\right)^{-\alpha}\left(\left|z_{2}-\xi\right|^{2}+t_{2}^{2}\right)^{-\beta} d \xi
$$

with $z_{i} \in R^{2}$, and $t_{i}>0$. The exponents are complex numbers, and the convergence properties of this integral will turn out in the course of the calculation. We shift the integration variable, and use a Feynman parametrization for both factors, $x^{-\gamma}=\Gamma^{-1}(\gamma) \int_{0}^{\infty} s^{\gamma-1} e^{-x s} d s$. Then the $\xi$-integration gets Gaussian, and we obtain

$$
C_{\alpha, \beta}=\frac{\pi}{\Gamma(\alpha) \Gamma(\beta)} \int_{0}^{\infty} \int_{0}^{\infty} d s_{1} d s_{2} \frac{s_{1}^{\alpha-1} s_{2}^{\beta-1}}{s_{1}+s_{2}} \exp \left(-\frac{\left|z_{1}-z_{2}\right|^{2} s_{1} s_{2}}{s_{1}+s_{2}}-s_{1} t_{1}^{2}-s_{2} t_{2}^{2}\right) .
$$

Next we perform the transformation $s_{1}=u s, s_{2}=(1-u) s, 0 \leqslant s \leqslant \infty, 0 \leqslant u \leqslant 1$, with the Jacobian $s=s_{1}+s_{2}$. The $s$-integration is trivial, and we are left with

$$
\begin{aligned}
C_{\alpha, \beta}= & \pi \Gamma(\alpha+\beta-1) \Gamma^{-1}(\alpha) \Gamma^{-1}(\beta) \int_{0}^{1} d u u^{\alpha-1}(1-u)^{\beta-1}\left(\left|z_{1}-z_{2}\right|^{2} u(1-u)\right. \\
& \left.+u t_{1}^{2}+(1-u) t_{2}^{2}\right)^{1-(\alpha+\beta)} .
\end{aligned}
$$

We may write, by means of the transformation $y=\left(t_{1} / t_{2}\right) u(1-u)^{-1}$,

$$
\begin{aligned}
C_{\alpha, \beta} t_{1}^{\alpha} t_{2}^{\beta} & =\int_{R^{2}} P^{\alpha}\left(z_{1}, t_{1} ; \xi\right) P^{\beta}\left(z_{2}, t_{2} ; \xi\right) d \xi \\
& =\frac{\pi \Gamma(\alpha+\beta-1)}{\Gamma(\alpha) \Gamma(\beta)} t_{2}^{2-\alpha-\beta} \int_{0}^{\infty} \frac{d y y^{\alpha-1}\left(1+\left(t_{2} / t_{1}\right) y\right)^{\alpha+\beta-2}}{\left(1+2(1+2 L) y+y^{2}\right)^{\alpha+\beta-1}},
\end{aligned}
$$

with the $H^{3}$-Poisson kernel and the Selberg point-pair invariant,

$$
P(z, t ; \xi):=\frac{t}{|z-\xi|^{2}+t^{2}}, L\left(z_{1}, t_{1} ; z_{2}, t_{2}\right):=\frac{\left|z_{1}-z_{2}\right|^{2}+\left(t_{1}-t_{2}\right)^{2}}{4 t_{1} t_{2}},
$$

respectively. We specify $\alpha+\beta=2$, and writing $L$ for $L\left(z_{1}, t_{1} ; z_{2}, t_{2}\right)$, we find

$$
C_{\alpha, 2-\alpha} t_{1}^{\alpha} t_{2}^{2-\alpha}=\frac{\pi}{4} \frac{1}{1-\alpha} \frac{\rho_{+}^{1-\alpha}-\rho_{-}^{1-\alpha}}{\sqrt{L(1+L)}},
$$

with $\rho_{ \pm}:=1+2 L \pm 2 \sqrt{L(L+1)}$, cf. (3.7). Finally, we put $\alpha=1+i s$, and obtain the kernel (3.14) as 


$$
k(s, d):=C_{1+i s, 1-i s} t_{1}^{1+i s} t_{2}^{1-i s}=\pi \frac{\sin (s d)}{s \sinh (d)},
$$

with the hyperbolic distance function $d=\log \rho_{+}(L)$.

The scalar orthogonality relation for the longitudinal time component in (2.16) can be derived from

$$
\begin{aligned}
\widetilde{\delta}\left(\xi, \xi^{\prime} ; \alpha, \beta ; \varepsilon\right)= & \int_{0}^{\infty} d t C_{\alpha, \beta}\left(\xi, t ; \xi^{\prime}, t\right) t^{\alpha+\beta-3+\varepsilon} \\
= & \frac{\pi}{2} \frac{\Gamma(\alpha+\beta-1)}{\Gamma(\alpha) \Gamma(\beta)}\left|\xi-\xi^{\prime}\right|^{\varepsilon-\alpha-\beta} \\
& \times B\left(\frac{\alpha-\beta+\varepsilon}{2}, \frac{\beta-\alpha+\varepsilon}{2}\right) B\left(\frac{\alpha+\beta-2+\varepsilon}{2}, \frac{\alpha+\beta-\varepsilon}{2}\right) .
\end{aligned}
$$

We used here the representation (A3) and interchanged integrations. We put $\alpha=1+i s, \beta=1$ - it, and write

$$
\delta\left(\xi, \xi^{\prime} ; s, t\right):=\widetilde{\delta}\left(\xi, \xi^{\prime} ; 1+i s, 1-i t ; \varepsilon\right)=\frac{\pi}{s^{2}}\left|\xi-\xi^{\prime}\right|^{\varepsilon-2+i(t-s)} \frac{i \varepsilon}{t-s+i \varepsilon}
$$

where we have dropped higher orders in $\varepsilon . \delta\left(\xi, \xi^{\prime} ; s, t\right)$ is a distribution concentrated at $\xi=\xi^{\prime}$, $t=s$. Next we note two $\varepsilon$-representations of the Dirac $\delta$-function, as well as two other well known formulas frequently used in this paper, cf. Ref. 25,

$$
\begin{gathered}
\delta(x)=\frac{1}{\pi} \frac{\varepsilon}{x^{2}+\varepsilon^{2}}, \quad \mp \pi i \delta(x)=\frac{1}{x \pm i \varepsilon}-P \frac{1}{x}, \quad \pm \pi i \theta(-x)=\log (x \pm i \varepsilon)-\log |x|, \\
\delta\left(d^{2}-\tau^{2}\right)=(2 d)^{-1}(\delta(d-\tau)+\delta(d+\tau)) .
\end{gathered}
$$

By integrating over a test function in $R^{2} \times R^{+}$, and making use of the first formula in (A10), one can easily show that

$$
\delta\left(\xi, \xi^{\prime} ; s, t\right)=2 \pi^{3} s^{-2} \delta\left(\xi-\xi^{\prime}\right) \delta(s-t)
$$

$\delta\left(\xi-\xi^{\prime}\right)$ is the Dirac function in $R^{2}$. The orthogonality relation for the time component of the vectors (2.16), which satisfy the homogeneous scalar Eq. (2.5), now follows from (A1), (A8), (A9), and (A11),

$$
\int_{H^{3}} d V_{H^{3}} P^{1+i s}(z, t ; \xi) P^{1-i t}\left(z, t ; \xi^{\prime}\right)=2 \pi^{3} s^{-2} \delta\left(\xi-\xi^{\prime}\right) \delta(s-t) .
$$

The $H^{3}$-volume element is $d V_{H^{3}}=t^{-3} d t d z_{1} d z_{2}$. Accordingly, we find the spectral measure

$$
d \sigma(\xi, s):=\left(2 \pi^{3}\right)^{-1} s^{2} e^{-\varepsilon s} d \xi d s
$$

on $R^{2} \times R^{+}$. A convergence factor $e^{-\varepsilon s}$ is included to obtain an $\varepsilon$-representation of the $\delta$-function via the completeness relation; a principal value as regularization is likewise possible. ${ }^{10}$ The eigenfunctions $P^{1+i s}$ satisfy $-\Delta_{H^{3}} P^{1+i s}=\left(s^{2}+1\right) P^{1+i s}$.

Once the spectral measure is found, one can write down the completeness relation,

$$
\int_{R^{2} \times R^{+}} d \sigma(\xi, s) P^{1+i s}(z, t ; \xi) P^{1-i s}\left(z_{0}, t_{0} ; \xi\right)=\delta_{H^{3}}\left(z, t ; z_{0}, t_{0}\right)
$$


or, more explicitly,

$$
\delta(L, \varepsilon):=\left(2 \pi^{3}\right)^{-1} \int_{0}^{\infty} k(s, d(L)) e^{-\varepsilon s} s^{2} d s=\frac{1}{2 \pi^{2}} \frac{\log \rho_{+}}{\sqrt{L(1+L)}} \frac{\varepsilon}{\left(\log ^{2} \rho_{+}+\varepsilon^{2}\right)^{2}} .
$$

If we expand in $\sqrt{L}$ and drop terms vanishing for $\varepsilon \rightarrow 0$, we find an $\varepsilon$-representation of the $\delta$-function in hyperbolic space,

$$
\delta\left(L\left(z, t ; z_{0}, t_{0}\right), \varepsilon\right)=\frac{1}{\pi^{2}} \frac{\varepsilon}{\left(\varepsilon^{2}+4 L\right)^{2}}=\delta_{H^{3}}\left(z, t ; z_{0}, t_{0}\right),
$$

with $L$ as in (A5). This can easily be checked by integration with a test function, preferably in the $B^{3}$-model; $\delta_{B^{3}}\left(\mathbf{x} ; \mathbf{x}_{0}\right)$ is obtained by replacing in (A16) $L\left(z, t ; z_{0}, t_{0}\right)$ by the $B^{3}$-invariant $L\left(\mathbf{x}, \mathbf{x}_{0}\right)$ as defined in (3.5). We may of course assume $\mathbf{x}_{0}=0$, because $L$ is invariant with respect to the symmetry group of $B^{3}$, which includes the transformations (3.4), and then use polar coordinates and a Taylor expansion of the test function $f(\mathbf{x})$ in $\int_{B^{3}} d V_{B^{3}} f(\mathbf{x}) \delta_{B^{3}}\left(\mathbf{x} ; \mathbf{x}_{0}\right)=f\left(\mathbf{x}_{0}\right)$; the $B^{3}$-volume element is $d V_{B^{3}}=8\left(1-|\mathbf{x}|^{2}\right)^{-3} d^{3} \mathbf{x}$.

\section{APPENDIX B: TRANSVERSAL AND LONGITUDINAL SPECTRAL MEASURES FOR THE SPATIAL COMPONENT OF THE PROCA EQUATION}

To calculate the space component of the resolvent kernel, we need the spectral measure in the completeness relation for the eigenvectors $\mathbf{a}^{T_{1,2}, L}$, cf. (2.18) and (2.19), which can be extracted from the orthogonality relation derived in this appendix. The basic integral in this relation was already calculated in (A8),

$$
\begin{aligned}
\int_{H^{3}} P^{\alpha}(z, t ; \xi) P^{\beta}\left(z, t ; \xi^{\prime}\right) d z t^{\gamma} d t= & \frac{\pi}{2} \frac{\left|\xi-\xi^{\prime}\right|^{3-\alpha-\beta+\gamma}}{\Gamma(\alpha) \Gamma(\beta) \Gamma(3+\gamma)} \Gamma\left(\frac{\alpha-\beta+\gamma+3}{2}\right) \Gamma\left(\frac{3-\alpha+\beta+\gamma}{2}\right) \\
& \times \Gamma\left(\frac{\alpha+\beta+\gamma+1}{2}\right) \Gamma\left(\frac{\alpha+\beta-\gamma-3}{2}\right),
\end{aligned}
$$

with the Poisson kernel $P$ as defined in (A5) and arbitrary complex numbers $\alpha, \beta$, and $\gamma$. (At this point there is no need to discuss convergence, though this is not hard to do; $H^{3}$ means here of course $R^{2} \times R^{+}$.) The orthogonality relation can be derived by differentiating this formula with respect to $\xi:=\left(\xi_{1}, \xi_{2}\right)$ and $\xi^{\prime}:=\left(\xi_{1}^{\prime}, \xi_{2}^{\prime}\right)$. Defining $e:=\varepsilon+i\left(s^{\prime}-s\right)$, we find in leading asymptotic order, for $s^{\prime} \rightarrow s$ and $\varepsilon \rightarrow 0$,

$$
\begin{gathered}
\int_{H^{3}} P^{i s}(z, t ; \xi) P^{-i s^{\prime}}\left(z, t ; \xi^{\prime}\right) d z t^{-1+\varepsilon} d t \sim \frac{2 \pi s^{2}}{e \bar{e}(1+e / 2)}\left|\xi-\xi^{\prime}\right|^{2+e}, \\
\int_{H^{3}} P^{1+i s} P^{1-i s^{\prime}} d z t^{-1+\varepsilon} d t \sim-\frac{\pi}{e}\left|\xi-\xi^{\prime}\right|^{e} \\
\int_{H^{3}} P^{2+i s} P^{2-i s^{\prime}} d z t^{-1+\varepsilon} d t \sim \frac{\pi}{2\left(s^{2}+1\right)}(1+\bar{e} / 2)\left|\xi-\xi^{\prime}\right|^{-2+e}, \\
\int_{H^{3}} P^{2+i s} P^{-i s^{\prime}} d z t^{-1+\varepsilon} d t=\int P^{i s} P^{2-i s^{\prime}} d z t^{-1+\varepsilon} d t \sim-\frac{\pi}{e}\left|\xi-\xi^{\prime}\right|^{e},
\end{gathered}
$$

which readily follows from (B1), by expanding the $\Gamma$-functions.

In the eigenvectors (2.18), we may replace the $z$-differentiations by $\xi$-differentiations, $\partial / \partial z_{i}$ $\rightarrow-\partial / \partial \xi_{i}$. [Note that in this appendix $z=\left(z_{1}, z_{2}\right)$, whereas in Appendix A the $z_{i}$ are themselves complex.] We define $D_{i j}:=\partial^{2} / \partial \xi_{i}^{2}-\partial^{2} / \partial \xi_{j}^{2}$, and analogously $D_{i j}^{\prime}$ for $\xi^{\prime}$, so that 


$$
\begin{gathered}
D_{12} D_{12}^{\prime}\left|\xi-\xi^{\prime}\right|^{2+e}=(e+2) e\left[4(e-1)\left|\xi-\xi^{\prime}\right|^{e-2}+\left(\left(\xi_{1}-\xi_{1}^{\prime}\right)^{2}\right.\right. \\
\left.\left.-\left(\xi_{2}-\xi_{2}^{\prime}\right)^{2}\right)^{2}(e-2)(e-4)\left|\xi-\xi^{\prime}\right|^{e-6}\right], \\
\frac{\partial^{4}}{\partial \xi_{1} \partial \xi_{2} \partial \xi_{1}^{\prime} \partial \xi_{2}^{\prime}}\left|\xi-\xi^{\prime}\right|^{2+e}=(e+2) e\left((e-1)\left|\xi-\xi^{\prime}\right|^{e-2}+\left(\xi_{1}-\xi_{1}^{\prime}\right)^{2}\right. \\
\left.\times\left(\xi_{2}-\xi_{2}^{\prime}\right)^{2}(e-2)(e-4)\left|\xi-\xi^{\prime}\right|^{e-6}\right), \\
D_{12}\left|\xi-\xi^{\prime}\right|^{e}=D_{12}^{\prime}\left|\xi-\xi^{\prime}\right|^{e}=e(e-2)\left(\left(\xi_{1}-\xi_{1}^{\prime}\right)^{2}-\left(\xi_{2}-\xi_{2}^{\prime}\right)^{2}\right)\left|\xi-\xi^{\prime}\right|^{e-4}, \\
\frac{\partial^{2}}{\partial \xi_{1} \partial \xi_{1}^{\prime}}\left|\xi-\xi^{\prime}\right|^{e}=-e\left|\xi-\xi^{\prime}\right|^{e-2}-e(e-2)\left(\xi_{1}-\xi_{1}^{\prime}\right)^{2}\left|\xi-\xi^{\prime}\right|^{e-4} .
\end{gathered}
$$

We thus find, by combining (2.18), (B2), and (B3),

$$
\begin{aligned}
& \int_{H^{3}} \mathbf{a}^{T_{1}}(s, \xi) \cdot \overline{\mathbf{a}^{T_{1}}\left(s^{\prime}, \xi^{\prime}\right)} d z t^{-1+\varepsilon} d t \\
&=\frac{1}{(1+i s)\left(1-i s^{\prime}\right)}\left(\frac{1}{16 s s^{\prime}} D_{12} P^{i s}(\xi) D_{12}^{\prime} P^{-i s^{\prime}}\left(\xi^{\prime}\right)+(1+i s)\right. \\
& \times\left(1-i s^{\prime}\right) P^{2+i s}(\xi) P^{2-i s^{\prime}}\left(\xi^{\prime}\right)+\frac{1+i s}{4 i s^{\prime}} P^{2+i s}(\xi) D_{12}^{\prime} P^{-i s^{\prime}}\left(\xi^{\prime}\right) \\
&-\frac{1-i s^{\prime}}{4 i s} P^{2-i s^{\prime}}\left(\xi^{\prime}\right) D_{12} P^{i s}(\xi)+\frac{1}{4 s s^{\prime}} \frac{\partial^{2}}{\partial \xi_{1} \partial \xi_{2}} P^{i s}(\xi) \frac{\partial^{2}}{\partial \xi_{1}^{\prime} \partial \xi_{2}^{\prime}} P^{-i s^{\prime}}\left(\xi^{\prime}\right) \\
&\left.+\frac{\partial}{\partial \xi_{1}} P^{1+i s}(\xi) \frac{\partial}{\partial \xi_{1}^{\prime}} P^{1-i s^{\prime}}\left(\xi^{\prime}\right)\right) \\
&= \frac{\pi}{\left(s^{2}+1\right)} \frac{\varepsilon}{\bar{e}}\left|\xi-\xi^{\prime}\right|^{e-2}=\int \mathbf{a}^{T_{2}} \cdot \overline{\mathbf{a}^{T_{2}}} d z t^{-1+\varepsilon} d t .
\end{aligned}
$$

We dropped here the $(z, t)$-arguments in the eigenfunctions and Poisson kernels. The $t^{-1}$-factor in the integral derives from the hyperbolic metric $\gamma_{i j}=t^{-2} \delta_{i j}$, via $\gamma^{i j} a_{i} a_{j} \sqrt{\gamma}$, and the $\varepsilon$-regularization is used to obtain an $\varepsilon$-representation of the $\delta$-functions in the orthogonality relation. In fact, by integrating over a test function, one can readily show that

$$
(\varepsilon / \bar{e})\left|\xi-\xi^{\prime}\right|^{e-2}=2 \pi^{2} \delta\left(\xi-\xi^{\prime}\right) \delta\left(s-s^{\prime}\right),
$$

with $\bar{e}:=\varepsilon-i\left(s^{\prime}-s\right)$. Similarly, for the longitudinal vectors in (2.18),

$$
\begin{aligned}
\int_{H^{3}} \mathbf{a}^{L}(s, \xi) \cdot \overline{\mathbf{a}^{L}\left(s^{\prime}, \xi^{\prime}\right)} d z t^{-1+\varepsilon} d t \\
=\frac{1}{(1+i s)\left(1-i s^{\prime}\right)}\left(\frac{\partial}{\partial \xi_{1}} P^{1+i s}(\xi) \frac{\partial}{\partial \xi_{1}^{\prime}} P^{1-i s^{\prime}}\left(\xi^{\prime}\right)+\frac{\partial}{\partial \xi_{2}} P^{1+i s}(\xi) \frac{\partial}{\partial \xi_{2}^{\prime}} P^{1-i s^{\prime}}\left(\xi^{\prime}\right)\right) \\
\quad+\frac{1}{t^{2}} P^{1+i s}(\xi) P^{1-i s^{\prime}}\left(\xi^{\prime}\right)+4 P^{2+i s}(\xi) P^{2-i s^{\prime}}\left(\xi^{\prime}\right)-\frac{2}{t} P^{1+i s}(\xi) P^{2-i s^{\prime}}\left(\xi^{\prime}\right) \\
\quad-\frac{2}{t} P^{2+i s}(\xi) P^{1-i s^{\prime}}\left(\xi^{\prime}\right)=\frac{\pi}{s^{2}} \frac{\varepsilon}{\bar{e}}\left|\xi-\xi^{\prime}\right|^{e-2}
\end{aligned}
$$


Here we used (B2), (B3), and in addition,

$$
\begin{gathered}
\int_{H^{3}} P^{1+i s} P^{1-i s^{\prime}} d z t^{-3+\varepsilon} d t \sim \frac{\pi \varepsilon}{\bar{e} s s^{\prime}}\left|\xi-\xi^{\prime}\right|^{e-2}, \\
\int_{H^{3}} P^{1+i s} P^{2-i s^{\prime}} d z t^{-2+\varepsilon} d t \sim \frac{\pi}{2} \frac{\varepsilon}{\left(1-i s^{\prime}\right) i s^{\prime}}\left|\xi-\xi^{\prime}\right|^{e-2}, \\
\int_{H^{3}} P^{2+i s} P^{1-i s^{\prime}} d z t^{-2+\varepsilon} d t \sim-\frac{\pi}{2} \frac{\varepsilon}{(1+i s) i s}\left|\xi-\xi^{\prime}\right|^{e-2},
\end{gathered}
$$

which follows from (B1) for $s^{\prime} \rightarrow s$ and $\varepsilon \rightarrow 0$. Equations (B4) and (B6), with (B5) substituted, constitute the orthogonality relations for the vector fields (2.18), if complemented by $\mathbf{a}^{T_{i}}(z, t ; \xi, s) \cdot \mathbf{a}^{L}\left(z, t ; \xi^{\prime}, s^{\prime}\right) \equiv 0$. The orthogonality of the longitudinal and transversal subspaces can readily be checked via (2.17), or via (2.18) and (2.19) and the following identities for the Poisson kernel,

$$
\begin{gathered}
\frac{\partial^{2} P^{\alpha}}{\partial z_{1} \partial z_{2}}=\frac{\alpha}{1+\alpha} \frac{1}{P^{2+\alpha}} \frac{\partial P^{1+\alpha}}{\partial z_{1}} \frac{\partial P^{1+\alpha}}{\partial z_{2}}, \\
D_{i j} P^{\alpha}=\frac{\alpha}{1+\alpha} \frac{1}{P^{2+\alpha}}\left(\left(\frac{\partial P^{1+\alpha}}{\partial z_{i}}\right)^{2}-\left(\frac{\partial P^{1+\alpha}}{\partial z_{j}}\right)^{2}\right), \\
\left(\frac{\partial P^{1+\alpha}}{\partial z_{1}}\right)^{2}+\left(\frac{\partial P^{1+\alpha}}{\partial z_{2}}\right)^{2}=4(1+\alpha)^{2} P^{4+2 \alpha}\left(\frac{1}{t P}-1\right), \\
\frac{\partial P^{1+\alpha}}{\partial t} \frac{\partial P^{1+\beta}}{\partial t}+\frac{\partial P^{1+\alpha}}{\partial z_{i}} \frac{\partial P^{1+\beta}}{\partial z_{i}}=(1+\alpha)(1+\beta) \frac{P^{2+\alpha+\beta}}{t^{2}}, \\
-\Delta_{H^{3}} P^{\alpha}(z, t ; \xi)=\alpha(2-\alpha) P^{\alpha}(z, t ; \xi) .
\end{gathered}
$$

$\alpha$ and $\beta$ are complex constants; the Laplace-Beltrami operator $\Delta_{H^{3}}$ is defined in (2.5), and the $D_{i j}$ are defined either with $z_{i}$-derivatives as in (2.18) or with $\xi_{i}$-derivatives as after (B2); summation over $i$ is implied in (B11).

The spectral measures for the transversal and longitudinal components can be read off from (B4)-(B6),

$$
d \sigma^{T}(\xi, s):=\frac{s^{2}+1}{2 \pi^{3}} e^{-\varepsilon s} d \xi d s, \quad d \sigma^{L}(\xi, s):=\frac{s^{2}}{2 \pi^{3}} e^{-\varepsilon s} d \xi d s
$$

the domain of integration is $R^{2} \times R^{+}$. As in (A13), we have included a convergence factor $e^{-\varepsilon s}$ needed in the completeness relation,

$$
\begin{aligned}
& \int_{R^{2} \times R^{+}} \sum_{k=1,2} \mathbf{a}_{i}^{T_{k}}(z, t ; \xi, s) \overline{\mathbf{a}_{j}^{T_{k}}\left(z^{\prime}, t^{\prime} ; \xi, s\right)} d \sigma^{T}(\xi, s) \\
& \quad+\int_{R^{2} \times R^{+}} \mathbf{a}_{i}^{L}(z, t ; \xi, s) \overline{\mathbf{a}_{j}^{L}\left(z^{\prime}, t^{\prime} ; \xi, s\right)} d \sigma^{L}(\xi, s)=\gamma_{i j} \delta_{H^{3}}\left(z, t ; z^{\prime}, t^{\prime}\right),
\end{aligned}
$$

where $\delta_{H^{3}}$ denotes the $\delta$-function in $H^{3}$, as defined in (A16). A check of this relation, by explicit calculation of the integrals, is given at the end of Sec. IV. 


\section{APPENDIX C: MATRIX ELEMENTS APPEARING IN THE SPATIAL RESOLVENT KERNEL}

We will calculate the matrix elements (4.4), that is $C_{i j}^{T_{k}}:=\int_{R^{2}} \mathbf{a}_{i}{ }_{T_{k}} \overline{\mathbf{a}_{j} T_{k}} d \xi, C_{i j}^{T}:=C_{i j}^{T_{1}}+C_{i j}^{T_{2}}$, and $C_{i j}^{L}:=\int_{R^{2}} \mathbf{a}_{i}^{L} \overline{\mathbf{a}_{j}^{L}} d \xi$, with eigenfunctions as defined in (2.18) and (2.19), at $z=z^{\prime}=0$. We consider, at first for arbitrary $z$ and $z^{\prime}$, cf. (A4),

$$
\begin{aligned}
K\left(a, b ; L ; t, t^{\prime}\right) & :=\int_{R^{2}} P^{a}(z, t ; \xi) P^{b}\left(z^{\prime}, t^{\prime} ; \xi\right) d \xi \\
& =\frac{\pi \Gamma(a+b-1)}{\Gamma(a) \Gamma(b)} t^{\prime 2-a-b} \int_{0}^{\infty} \frac{d y y^{a-1}\left(1+\left(t^{\prime} / t\right) y\right)^{a+b-2}}{\left(1+2(1+2 L) y+y^{2}\right)^{a+b-1}},
\end{aligned}
$$

with the $H^{3}$-point-pair invariant $L\left(z, t ; z^{\prime}, t^{\prime}\right)$ as in (3.11), and define

$$
K(a, b):=\left.K\left(a, b ; L ; t, t^{\prime}\right)\right|_{z=z^{\prime}=0}, \quad K^{\prime}(a, b):=\partial K\left(a, b ; L ; t, t^{\prime}\right) /\left.\partial L\right|_{z=z^{\prime}=0},
$$

and analogously $K^{\prime \prime}(a, b)$, where $a$ and $b$ are arbitrary complex numbers, so that the integrals converge. It is understood that the $L$-differentiation is carried out before the $y$-integration in $(\mathrm{C} 1)$, to take care of the poles of $\Gamma(a+b-1)$. In the following, we put $\beta:=t^{\prime} / t$, so that, at $z=z^{\prime}$ $=0, \quad 1+2(1+2 L) y+y^{2}=(1+\beta y)\left(1+\beta^{-1} y\right)$. We also introduce the shortcuts $L_{, i}$ $=\partial L /\left.\partial z_{i}\right|_{z=z^{\prime}=0}$ and $L_{, i^{\prime}}=\partial L /\left.\partial z_{i}^{\prime}\right|_{z=z^{\prime}=0}$, with $z=:\left(z_{1}, z_{2}\right)$ and $z^{\prime}=:\left(z_{1}^{\prime}, z_{2}^{\prime}\right)$, and analogously for higher order derivatives. Evidently, $z_{1,2}$-derivatives of $K\left(a, b ; L ; t, t^{\prime}\right)$ at $z=z^{\prime}=0$ are obtained via $\partial K / \partial z_{i}=: K_{, i}=K^{\prime}(a, b) L_{, i}$, etc. We will only use $z_{1,2}$ and $z_{1,2}^{\prime}$-derivatives, always at $z=z^{\prime}=0 ; t$ and $t^{\prime}$-derivatives are not needed in the following. The only nonvanishing derivatives of $L$ are $L_{, i, j}=\left(2 t t^{\prime}\right)^{-1} \delta_{i j}, L_{, i, j^{\prime}}=-\left(2 t t^{\prime}\right)^{-1} \delta_{i j^{\prime}}$, and $L_{, i^{\prime}, j^{\prime}}=\left(2 t t^{\prime}\right)^{-1} \delta_{i^{\prime} j^{\prime}}$. Accordingly, the only nonvanishing $z_{1,2}$-derivatives of $K\left(a, b ; L ; t, t^{\prime}\right)$ at $z=z^{\prime}=0$ are, up to the fourth order

$$
\begin{gathered}
K_{, 1,1}(a, b)=K_{, 2,2}=\left(2 t t^{\prime}\right)^{-1} K^{\prime}(a, b), \\
K_{, 1,1,2,2}=\left(2 t t^{\prime}\right)^{-2} K^{\prime \prime}(a, b), \quad K_{, 1,1,1,1}=K_{, 2,2,2,2}=3 K_{, 1,1,2,2} .
\end{gathered}
$$

The indicated indices may of course be permuted. An index may also be replaced by the same primed index; in this case a minus sign has to be added on the right-hand side, e.g., $K_{, 1,1^{\prime}, 2,2}$ $=-K_{, 1,1,2,2}, K_{, 1,1^{\prime}, 2^{\prime}, 2}=K_{, 1,1,2,2}$, etc., since $\partial / \partial z_{i}=-\partial / \partial z_{i}^{\prime}$ when applied to $L$.

With these preparations, the matrix elements (4.4) can be readily calculated. We use the representation (2.18), (2.19) for the eigenfunctions, interchange differentiations, and integrations, and do some bookkeeping by means of $(\mathrm{C} 1)-(\mathrm{C} 3)$. In this way we find the nonvanishing matrix elements as

$$
\begin{gathered}
C_{11}^{T_{1}}=\frac{1}{16 s^{2}\left(s^{2}+1\right)} \frac{K^{\prime \prime}(0,0)}{t^{2} t^{\prime 2}}+K(2,2), \\
C_{22}^{T_{1}}=\frac{1}{16 s^{2}\left(s^{2}+1\right)} \frac{K^{\prime \prime}(0,0)}{t^{2} t^{\prime 2}}, \quad C_{33}^{T_{1}}=\frac{-1}{2\left(s^{2}+1\right)} \frac{K^{\prime}(1,1)}{t t^{\prime}}, \\
C_{11}^{T_{2}}=C_{22}^{T_{1}}, \quad C_{22}^{T_{2}}=C_{11}^{T_{1}}, \quad C_{11}^{L}=C_{22}^{L}=C_{33}^{T_{2}}=C_{33}^{T_{1}}, \\
C_{33}^{L}=\frac{K(1,1)}{t t^{\prime}}+4 K(2,2)-2 \frac{K(1,2)}{t}-2 \frac{K(2,1)}{t^{\prime}} .
\end{gathered}
$$

Next we define

$$
\kappa(l, m, n ; s):=\int_{0}^{\infty} \frac{d y y^{l+i s}}{(1+\beta y)^{m}\left(1+\beta^{-1} y\right)^{n}},
$$


with $\beta:=t^{\prime} / t$, so that

$$
\begin{gathered}
K(1,1)=\pi \frac{\kappa(0,1,1 ; s)}{\Gamma(1+i s) \Gamma(1-i s)}, \quad K(1,2)=\frac{1}{t^{\prime}} \frac{\pi \kappa(0,1,2 ; s)}{\Gamma(1+i s) \Gamma(2-i s)}, \\
K(2,1)=\frac{1}{t^{\prime}} \frac{\pi \kappa(1,1,2 ; s)}{\Gamma(2+i s) \Gamma(1-i s)}, \quad K(2,2)=\frac{1}{t^{\prime 2}} \frac{2 \pi \kappa(1,1,3 ; s)}{\Gamma(2+i s) \Gamma(2-i s)}, \\
K^{\prime}(1,1)=\frac{-4 \pi \kappa(1,2,2 ; s)}{\Gamma(1+i s) \Gamma(1-i s)}, \quad K^{\prime \prime}(0,0)=t^{\prime 2} \frac{16 \pi \kappa(1,3,1 ; s)}{\Gamma(i s) \Gamma(-i s)} .
\end{gathered}
$$

As a consistency check, we note the symmetry

$$
K\left(2,2 ; \beta \rightarrow \beta^{-1}\right)=\frac{K^{\prime \prime}(0,0 ; \beta)}{8 t^{\prime 4} s^{2}\left(s^{2}+1\right)} .
$$

The integrals (C6) are elementary,

$$
\begin{gathered}
K(1,1)=\frac{\pi}{i s} \frac{\beta}{\beta^{2}-1}\left(\beta^{i s}-\beta^{-i s}\right), \\
K(1,2)=\frac{\pi}{i s(1-i s)} \frac{\beta}{\left(\beta^{2}-1\right)^{2}} \frac{1}{t^{\prime}}\left(\beta^{2+i s}-\beta^{2-i s}-i s \beta^{i s}\left(\beta^{2}-1\right)\right), \\
K(2,1)=-\frac{\pi}{i s(1+i s)} \frac{\beta^{2}}{\left(\beta^{2}-1\right)^{2}} \frac{1}{t^{\prime}}\left(\beta^{i s}-\beta^{-i s}-i s \beta^{i s}\left(\beta^{2}-1\right)\right), \\
K(2,2)=-\frac{2 \pi}{\left(s^{2}+1\right)} \frac{\beta^{4}}{\left(\beta^{2}-1\right)^{3}} \frac{1}{t^{\prime 2}} \frac{\beta^{i s}-\beta^{-i s}}{i s}+\frac{\pi}{\left(s^{2}+1\right)} \frac{\beta^{2+i s}}{\left(\beta^{2}-1\right)^{2}} \frac{1}{t^{\prime 2}}\left(\left(\beta^{2}+1\right)-i s\left(\beta^{2}-1\right)\right), \\
K^{\prime}(1,1)=-4 \pi \frac{\beta^{2}\left(\beta^{2}+1\right)}{\left(\beta^{2}-1\right)^{3}} \frac{\beta^{i s}-\beta^{-i s}}{i s}+\frac{4 \pi \beta^{2}}{\left(\beta^{2}-1\right)^{2}}\left(\beta^{i s}+\beta^{-i s}\right), \\
K^{\prime \prime}(0,0)=\frac{16 \pi \beta^{2}}{\left(\beta^{2}-1\right)^{3}} t^{\prime 2} i s\left(\beta^{i s}-\beta^{-i s}\right)+\frac{8 \pi s^{2} \beta^{-i s}}{\left(\beta^{2}-1\right)^{2}} t^{\prime 2}\left(\left(\beta^{2}+1\right)+i s\left(\beta^{2}-1\right)\right) .
\end{gathered}
$$

Collecting terms, we obtain the nonvanishing matrix elements $C_{i j}^{T, L}$ in (4.4) as

$$
\begin{aligned}
C_{11}^{T}=C_{22}^{T}= & \frac{\pi \beta}{\left(\beta^{2}-1\right)^{3}\left(s^{2}+1\right)} \frac{1}{t t^{\prime}} \\
& \times\left(-4 \beta^{2} \frac{\beta^{i s}-\beta^{-i s}}{i s}+\left(\beta^{4}-1\right)\left(\beta^{i s}+\beta^{-i s}\right)-\left(\beta^{2}-1\right)^{2} i s\left(\beta^{i s}-\beta^{-i s}\right)\right), \\
C_{33}^{T}= & C_{11}^{L}=C_{22}^{L}=\frac{2 \pi \beta^{2}}{\left(\beta^{2}-1\right)^{3}\left(s^{2}+1\right)} \frac{1}{t t^{\prime}}\left(\left(\beta^{2}+1\right) \frac{\beta^{i s}-\beta^{-i s}}{i s}-\left(\beta^{2}-1\right)\left(\beta^{i s}+\beta^{-i s}\right)\right), \\
C_{33}^{L}= & \frac{\pi \beta}{\left(\beta^{2}-1\right)^{3}\left(s^{2}+1\right)} \frac{1}{t t^{\prime}} \\
& \times\left(-\left(\beta^{4}+6 \beta^{2}+1\right) \frac{\beta^{i s}-\beta^{-i s}}{i s}-\left(\beta^{2}-1\right)^{2} i s\left(\beta^{i s}-\beta^{-i s}\right)+2\left(\beta^{4}-1\right)\left(\beta^{i s}+\beta^{-i s}\right)\right) .
\end{aligned}
$$


What remains is to substitute (C8) into the resolvent kernel (4.1) and (4.2), and to perform the $s$-integrations, which are likewise elementary, cf. (4.5)-(4.11).

${ }^{1}$ G. W. Gibbons, in General Relativity: An Einstein Centenary Survey, edited by S. W. Hawking and W. Israel (Cambridge University Press, Cambridge, 1979).

${ }^{2}$ N. D. Birrell and P. C. W. Davies, Quantum Fields in Curved Space (Cambridge University Press, Cambridge, 1994).

${ }^{3}$ J. A. Wheeler and R. P. Feynman, Rev. Mod. Phys. 17, 157 (1945).

${ }^{4}$ R. Tomaschitz, Class. Quantum Grav. 18, 4395 (2001).

${ }^{5}$ L. V. Ahlfors, Möbius Transformations in Several Dimensions, Lecture Notes (University of Minnesota, Minneapolis, 1981).

${ }^{6}$ S. J. Patterson, in Analytical and Geometrical Aspects of Hyperbolic Space, edited by D. B. A. Epstein (Cambridge University Press, London, 1987).

${ }^{7}$ D. A. Hejhal, The Selberg Trace Formula for PSL (2, R) (Springer, New York, 1976), Vol. 1, LNM 548.

${ }^{8}$ D. A. Hejhal, The Selberg Trace Formula for $\operatorname{PSL}(2, R)$ (Springer, New York, 1983), Vol. 2, LNM 1001.

${ }^{9}$ J. Elstrodt, F. Grunewald, and J. Mennicke, Groups Acting on Hyperbolic Space: Harmonic Analysis and Number Theory (Springer, Berlin, 1998).

${ }^{10}$ N. Mandouvalos, Proc. London Math. Soc. 57, 209 (1988).

${ }^{11}$ A. S. Goldhaber and M. M. Nieto, Rev. Mod. Phys. 43, 277 (1971).

${ }^{12}$ A. E. Beardon, The Geometry of Discrete Groups (Springer, New York, 1983).

${ }^{13}$ R. Tomaschitz, J. Math. Phys. 34, 3133 (1993).

${ }^{14}$ N. N. Bogoliubov and D. V. Shirkov, Introduction to the Theory of Quantized Fields (Wiley, New York, 1980).

${ }^{15}$ G. N. Watson, A Treatise on the Theory of Bessel Functions (Cambridge University Press, Cambridge, 1996).

${ }^{16} \mathrm{~F}$. Bloch and A. Nordsieck, Phys. Rev. 52, 54 (1937).

${ }^{17}$ R. Tomaschitz, Class. Quantum Grav. 16, 3349 (1999).

${ }^{18}$ S. J. Paterson, in Number Theory, Trace Formulas, and Discrete Groups, edited by K. E. Aubert (Academic, Boston, 1989).

${ }^{19}$ S. L. Krushkal, B. N. Apanasov, and N. A. Grusevskii, Kleinian Groups and Uniformization in Examples and Problems (AMS, Providence, 1986).

${ }^{20}$ B. Maskit, Kleinian Groups (Springer, New York, 1986).

${ }^{21}$ R. Tomaschitz, Physica D 34, 42 (1989).

${ }^{22}$ J. Dodziuk and J. Jorgenson, Mem. Am. Math. Soc. 643, 1 (1998).

${ }^{23}$ K. Matsuzaki and M. Taniguchi, Hyperbolic Manifolds and Kleinian Groups (Clarendon, Oxford, 1998).

${ }^{24}$ M. Kapovich, Hyperbolic Manifolds and Discrete Groups (Birkhäuser, Boston, 2001)

${ }^{25}$ M. Gel'fand and G. E. Shilov, Generalized Functions (Academic, New York, 1972), Vol. 1. 